University of South Florida

DIGITAL COMMONS

Digital Commons @ University of

@ UNIVERSITY OF SOUTH FLORIDA

South Florida

Marine Science Faculty Publications

College of Marine Science

2020

\title{
Eddy-wave Duality in a Rotating Flow
}

Stefania Espa

Sapienza Università di Roma

Simon Cabanes

Sapienza Università di Roma

Gregory P. King

University of South Florida

Gabriella Nitto

Sapienza Università di Roma

Boris Galperin

University of South Florida, bgalperin@usf.edu

Follow this and additional works at: https://digitalcommons.usf.edu/msc_facpub

Part of the Life Sciences Commons

\section{Scholar Commons Citation}

Espa, Stefania; Cabanes, Simon; King, Gregory P.; Nitto, Gabriella; and Galperin, Boris, "Eddy-wave Duality in a Rotating Flow" (2020). Marine Science Faculty Publications. 1512.

https://digitalcommons.usf.edu/msc_facpub/1512

This Article is brought to you for free and open access by the College of Marine Science at Digital Commons @ University of South Florida. It has been accepted for inclusion in Marine Science Faculty Publications by an authorized administrator of Digital Commons @ University of South Florida. For more information, please contact digitalcommons@usf.edu. 


\section{Eddy-wave duality in a rotating flow $\circledast$}

Cite as: Phys. Fluids 32, 076604 (2020); https://doi.org/10.1063/5.0006206

Submitted: 29 February 2020 . Accepted: 18 June 2020 . Published Online: 08 July 2020

Stefania Espa, Simon Cabanes, (D) Gregory P. King, Gabriella Di Nitto, and (D) Boris Galperin

\section{COLLECTIONS}

EP This paper was selected as an Editor's Pick


\section{ARTICLES YOU MAY BE INTERESTED IN}

Can a toilet promote virus transmission? From a fluid dynamics perspective

Physics of Fluids 32, 065107 (2020); https://doi.org/10.1063/5.0013318

Statistical and modal analysis of surface pressure fluctuations in tornado-like vortices

Physics of Fluids 32, 075109 (2020); https://doi.org/10.1063/5.0012446

Visualizing the effectiveness of face masks in obstructing respiratory jets

Physics of Fluids 32, 061708 (2020); https://doi.org/10.1063/5.0016018

\section{Physics of Fluids}




\title{
Eddy-wave duality in a rotating flow $\oplus$
}

\author{
Cite as: Phys. Fluids 32, 076604 (2020); doi: 10.1063/5.0006206 \\ Submitted: 29 February 2020 - Accepted: 18 June 2020 • \\ Published Online: 8 July 2020
}

\begin{abstract}
Stefania Espa,, (D) Simon Cabanes, ${ }^{1, b)}$ Gregory P. King, ${ }^{2, c)}$ (D) Gabriella Di Nitto, ${ }^{1, d)}$ and Boris Galperin
AFFILIATIONS

${ }^{1}$ Department of Civil, Construction and Environmental Engineering, Sapienza Università di Roma, Via Eudossiana 18, 00184 Rome, Italy

${ }^{2}$ College of Marine Science, University of South Florida, St. Petersburg, Florida 33701, USA
\end{abstract}

\author{
a) Author to whom correspondence should be addressed: stefania.espa@uniroma1.it \\ b) Electronic mail: simon.cabanes@gmail.com \\ c) Electronic mail: gking.lisboa@outlook.com \\ d) Electronic mail: gabriella.dinitto@gmail.com \\ e) Electronic mail: bgalperin@usf.edu
}

\begin{abstract}
A series of experiments with rotating, electromagnetically forced, turbulent flows were carried out at the Sapienza University of Rome to investigate the eddy-wave duality in flows with a $\beta$-effect and the electromagnetic force acting in the westward direction. When the $\beta$-effect is significant, i.e., as in planetary atmospheric and oceanic circulations, nonlinear eddy/wave interactions facilitate flow self-organization into zonal patterns in which Rossby waves and westward propagating cyclonic and anticyclonic eddies coexist. Upon time averaging, eddies disappear and the flow pattern transforms into a system of alternating zonal jets. What is the relationship between eddies, jets, and Rossby waves? To address this issue, we designed a laboratory experiment in which a westward zonal flow is produced by applying an electromagnetic small-scale forcing to a thin layer of a rotating fluid. In order to investigate different levels of flow zonality and a wider range of zonal modes, we varied the forcing intensity and the area of the forced sector. The zonal flow evolves as a system of westward propagating, large scale, cyclonic, and anticyclonic eddies. The propagation speed of the traveling structures was calculated from the Hovmöller diagrams of both the streamfunction and the centroids of clusters of different types (cyclonic and anticyclonic eddy cores and saddle point neighborhoods) obtained via an Okubo-Weiss analysis. The results were compared with the theoretical phase speed of a Rossby wave. The correspondence between these two characteristics at the radius of maximum shear corresponding to the epicenter of the barotropic instability is quite good, particularly after including the radial variation of the zonal velocity in the $\beta$-term. It is concluded that the Rossby waves and eddies are inseparable as the former maintain the instability that sustains the latter. This symbiosis visually resembles the Rossby soliton.
\end{abstract}

Published under license by AIP Publishing. https://doi.org/10.1063/5.0006206

\section{INTRODUCTION}

Geophysical and planetary circulations exhibit a rich variety of zonal flows, waves, and eddies produced and maintained by various instabilities. Flow zonation is mainly due to the restoring force associated with the latitudinal variation of the Coriolis parameter through the potential vorticity (PV) conservation, the socalled $\beta$-effect, which tends to return a displaced fluid particle to its equilibrium latitude, giving rise to fluctuations around that latitude known as the Rossby waves. ${ }^{1}$ Zonal flows may be eastward, westward, or in alternating directions. Even though most terrestrial oceanic and atmospheric jets are eastward, the focus here will be on westward jets. The reasons for this choice will be discussed below.

The asymmetry in the Rossby wave propagation induces the asymmetry between the eastward and westward zonal flows; indeed, eastward jets are stable and sharp, while the westward jets are unstable and blunt. Westward jets are prone to barotropic and baroclinic instabilities leading to PV mixing (which flattens the PV profile across the jet) and eddy shedding. ${ }^{2}$ Once formed, the jets coexist with multiple eddies created and maintained by these instabilities, while the eddies, in turn, transfer energy back to the jets, thus inducing a continuous exchange of energy via upscale/downscale turbulent cascades. 
Several examples of these features emerge from giant planets' atmospheres whose dynamics are strongly affected by the existence of zonal jets. ${ }^{4-6}$ Almost all westward jets on Jupiter and Saturn are barotropically unstable ${ }^{7}$ and shed cyclonic/anticyclonic eddies of different shapes and sizes. For example, on Saturn, the $40^{\circ} \mathrm{N}$ westward jet becomes unstable on its southern flank and radiates trains of westward propagating cyclonic eddies referred to as the String of Pearls. ${ }^{7}$ In Jupiter's atmosphere, near $30^{\circ} \mathrm{N}$ is the so-called Jovian Ribbon, a dynamic structure associated with a westward jet that irregularly undulates in latitude. ${ }^{8}$ Figure 1 shows Jupiter's zonal wind profile and the locations where most eddies are produced.' This figure suggests that most of the eddies are produced in the westward jets close to points of maximum shear. Such features are also common to the westward south-equatorial currents in all oceans. Under the action of a seasonal forcing, these currents periodically become unstable and radiate waves known as Tropical Instability Waves (TIWs). ${ }^{10-13}$ TIWs are Rossby waves and can be seen in satellite imagery as westward-traveling undulations in the sea surface temperature fronts on either side of the equatorial cold tongue (Fig. 2). Intimately associated with TIWs are a train of westward propagating anticyclonic eddies called Tropical Instability Vortices (TIVs). TIWs/TIVs exert major controls on the ocean dynamics, thermodynamics, and biology on intraseasonal to seasonal time scales $^{14}$ and have been found to play an important role in the El Niño Southern Oscillation (ENSO) phenomenon.

In these contexts, a challenging issue is to understand the relationship between Rossby waves and eddies (cf., Ref. 16). Are the eddies created after the Rossby wave reaches a critical amplitude? Or

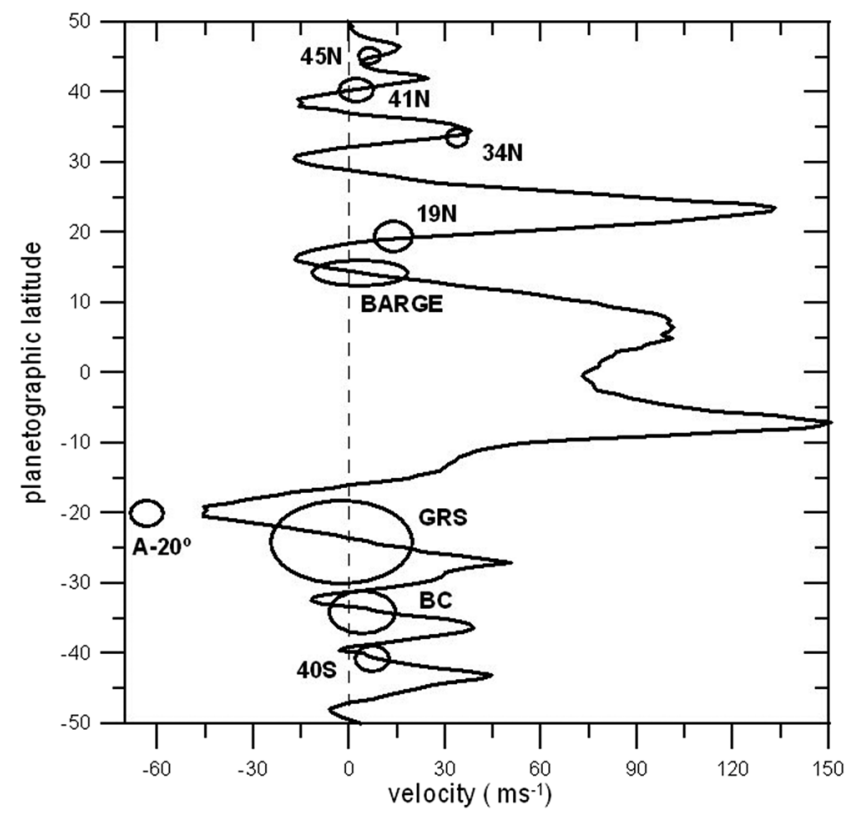

FIG. 1. Jupiter's zonal wind profile as measured by García-Melendo and SánchezLavega. ${ }^{9}$ Eddies are drawn as ovals scaled to their latitudinal size and centered on the latitudes where most are produced, which are close to points of maximum shear in the westward jets. In certain latitudes, there are "trains" of vortices (e.g., latitude $40^{\circ} \mathrm{S}$ ), but in others, they are isolated [e.g., the Great Red Spot (GRS)].

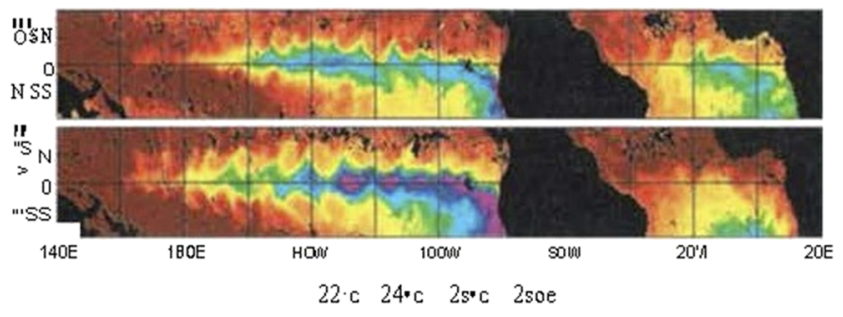

FIG. 2. Selected 3-day composite-average maps of sea surface temperature for the periods 11-13 July 1998 (upper) and 14-16 November 1998 (lower). Black areas represent land or rain contamination. Adapted from Chelton et al.

do the eddies and meandering jet together comprise the structure of a Rossby solitary wave? To investigate this issue, an experimental campaign was carried out in a rotating tank facility at the Sapienza University of Rome. Experiments were designed to excite a single westward jet in a barotropic rotating fluid using small-scale electromagnetic forcing. ${ }^{17,18}$ Velocity measurements show that the experiments produce a meandering jet and westward propagating eddies, similar to the geophysical examples above. After time averaging, the mean flow appears as a system of alternating zonal jets: a strong westward jet with a weak eastward jet on either side. The dispersion relation for Rossby waves with a mean zonal flow that changes in the radial direction is derived, and an expression for the phase speed is obtained that depends on the radial coordinate. Hovmöller diagrams of the streamfunction show propagating features that have a propagation velocity that corresponds to a linear Rossby wave with an azimuthal mode $m=1$. The best match between the propagation velocity measured in the experiments and the theoretical prediction occurs at the radius of maximum shear. Next, the Okubo-Weiss criterion is used to identify the neighborhoods of eddy centers (eddy cores) and the saddle points that separate adjacent eddies. Their propagation is tracked and phase speeds estimated using Hovmöller diagrams. The results show that the eddies and saddle points propagate as a single structure and, at the location of maximum shear, have a propagation velocity in agreement with a linear Rossby wave. The remainder of this paper is structured as follows. The experimental apparatus and methods are described in Sec. II. Theory, its application to the experiments, and the Okubo-Weiss methodology are described in Sec. III, and Sec. IV describes the results. We discuss and give our conclusions in Sec. V.

\section{THE EXPERIMENTAL FACILITY}

The two necessary pre-requisites making an experimental facility suitable for this study are (a) the $\beta$-effect and (b) the possibility of creating an easily controllable westward zonal jet. The experimental apparatus at the University of Rome satisfies both requirements ${ }^{18}$ (Fig. 1). As widely described in previous papers, ${ }^{19-23}$ the facility consists of a square tank placed on a rotating table, $1 \mathrm{~m}$ in diameter, spinning counter-clockwise to emulate flows in the Northern Hemisphere. The internal dimensions of the tank are $68 \times 68 \times 15 \mathrm{~cm}^{3}$. The working fluid is a thin layer of saline solution with a mean depth $H_{0}=4 \mathrm{~cm}$. When a fluid layer rotates its free surface, it assumes a parabolic shape, with a radial variation of the fluid depth, 


$$
H(r)=H_{0}-\frac{\Omega^{2} R^{2}}{4 g}+\left(\frac{\Omega^{2}}{2 g}\right) r^{2},
$$

where $r$ is the radial distance from the center of the tank and $g$ is the gravitational acceleration. The parabolic shape of the free surface is ideal for the formation of zonal jet flows as it induces a gradient of potential vorticity known as the $\beta$-effect. Note that the paraboloidal surface shape is the first-order Taylor expansion of the spherical curvature near the planetary polar region ${ }^{24}$ and yields a local $\beta$-plane approximation. $^{2}$

In our laboratory experiments, the rotation rate is $\Omega=3 \mathrm{rad} \mathrm{s}^{-1}$; the $\beta$-effect arises due to the cylindrically radial variations of the fluid layer depth and defined as a "topographic" $\beta=2 \Omega H^{-1} d H / d r$. We estimate $\beta$ at mid radius $R / 2$ that corresponds to

$$
\beta=\frac{\Omega^{3} R}{g H(R / 2)},
$$

with $R$ being the maximum radial distance. ${ }^{19,25}$ Being the point of the minimum fluid depth, the center of the tank represents the planetary pole, a pole in a planetary prototype, while the edge of the domain corresponds to lower latitudes. The zonal (azimuthal in polar coordinates) jet was produced using electromagnetic forcing ${ }^{17}$ on a solution of water and $\mathrm{NaCl}$; in particular, the electric current is assumed homogeneous and running only in the horizontal direction orthogonal to the electrodes $^{23}$ (Fig. 1), while the main component of the magnetic field is along the vertical direction. Thus, the Lorentz force acts in the horizontal direction perpendicular to both of these fields and may be positive or negative, dependent on the orientation of the magnet. ${ }^{26}$ The current was generated by two titanium electrodes placed inside the tank, along two opposite sidewalls, and connected to a power supply. When the fluid attained solid-body rotation, a constant voltage is applied to the electrodes. Circular permanent (neodymium) magnets were placed along arcs of $90^{\circ}$ or $180^{\circ}$ of the radius $r=17 \mathrm{~cm}$ and $0.5 \mathrm{~cm}$ underneath the tank bottom. All magnets had the same polarity such that we introduce a westward momentum to facilitate the formation of a westward zonal jet. Note that the stationary position of the magnets locked-in the location of the jet. ${ }^{18}$

The flow field was measured using the image analysis technique known as Feature Tracking (FT). ${ }^{27}$ To this aim, the fluid surface was seeded with styrene particles (mean diameter of $\sim 50 \mu \mathrm{m}$ ) and illuminated by two lateral lamps. A Toshiba CSB1100CL video camera co-rotating with the system and with its optical axis parallel to the rotation axis acquired the flow images of the tank top surface (image size: $1023 \times 1240$ pixels). FT is a multi-frame algorithm based on the assumption that, for short time intervals, particles' light intensity is conserved between one frame and the successive, as well as in both spatial directions; it allows us to reconstruct the velocity field in a Lagrangian framework from the acquired images. Basically, it considers a measure of correlation windows between successive frames and evaluates displacements by considering the best correspondence (in terms of a defined matching measure) of selected interrogation windows between subsequent images. Lagrangian velocities are obtained dividing the displacement by the time interval between two frames $\left(0.05 \mathrm{~s}\right.$ in this case). In these experiments, about $\mathrm{O}\left(10^{4}\right)$ features have been simultaneously traced. It is then possible to interpolate the sparse velocity vectors on a regular grid so to obtain the time sequence of the instantaneous Eulerian velocity fields.
The geometrical configuration of our setup suggests the use of the two-dimensional (2D) polar coordinate system $(r, \varphi)$. The azimuthal direction $\varphi$ identifies points with the same fluid depth (the so-called zonal direction) and at constant radius $r$. Due to PV conservation these points correspond to points with the same latitude on a rotating planet. The sparse velocities are then interpolated using a standard procedure onto a regular polar grid of 120 and 60 grid points in azimuth and radius, respectively. The radial and azimuthal velocity components are evaluated at each grid point; a more detailed description of the procedure can be found in Di Nitto, Espa, and Cenedese. ${ }^{2}$

We performed three experiments in the $90^{\circ}$ configuration (denoted as $1_{90}, 290$, and $3_{90}$ ), and three experiments in the $180^{\circ}$ configuration (denoted as $1_{180}, 2_{180}$, and $3_{180}$ ). For each configuration, we only vary the intensity $I$ of the electrical current applied to the saline solution from $2 \mathrm{~A}$ up to $6 \mathrm{~A}$, the experimental parameters are listed in Table I. The Rossby number $R o=U_{r m s} / 2 \Omega H_{O}$ is in the range of $\mathrm{O}\left(10^{-2}\right)-\mathrm{O}\left(10^{-3}\right)$, the Reynolds number $R e=U_{r m s} H_{O} / v$ is $\mathrm{O}\left(10^{2}\right)$, the Ekman number $E k=R o / R e$ is $\mathrm{O}\left(10^{-4}\right), U_{r m s}$ is the root mean square of the total velocity, and $v$ is the kinematic viscosity. As shown in Fig. 3 , in the $90^{\circ}$ arc experiments, the domain was divided into four $90^{\circ}$ sectors: in the clockwise direction, sector I is the forced region $\left(221^{\circ} \leq \varphi \leq 310^{\circ}\right)$ and sectors II-IV are the unforced sectors. For the $180^{\circ}$ arc experiments, the domain was divided into two sectors: the forced sector $\left(181^{\circ} \leq \varphi \leq 360^{\circ}\right)$ and the unforced one (Fig. 3).

Figure 4 shows the streamfunction $\psi$, instantaneous on the left and time-averaged on the right, taken from experiments 390 (top) and $3_{180}$ (bottom). The panels on the left show strong cyclonic and anticyclonic eddies propagating westward with a strong narrow meandering jet sandwiched between them. Due to conservation of angular momentum, there are also two weak satellite eastward jets that are difficult to make out in these figures. The time-averaged streamfunctions (right) are quite different as they show alternating zonal jets and no eddies at all. A similar picture emerges in observations of oceanic eddies where short-time flow images also show multiple westward propagating eddies, ${ }^{28}$ while time-averaged images show multiple alternating zonal jets (see Refs. 29 and 30). Note that similar to other experiments, ${ }^{31,32}$ the scales of the eddies and jets in the experiments exceed the forcing scales in the experiments, thus pointing to the action of an inverse energy cascade. Following the same procedure as in Ref. 18, we can show (not detailed here) that in our experiment, the energy of the turbulent inverse cascade relies on the well-known Kolmogorov-Kraichnan ${ }^{33}$ spectral law $\varepsilon^{2 / 3} k^{-5 / 3}$,

TABLE I. Parameters used in both the $S=90^{\circ}$ and $180^{\circ}$ experiments, the notation is the same as used in Ref. 17. The typical Rhines scale is calculated as $L_{R h}=\sqrt{2 U_{r m s} / \beta}$. We note $U_{r m s}$ the root mean square of the total velocity. The energy transfer rate $\varepsilon$ of the upscale energy cascade is estimated from a spectral decomposition of the streamfunction, as detailed in Ref. 18.

\begin{tabular}{lccccc}
\hline \hline Expt. & $I(\mathrm{~A})$ & $H(\mathrm{~cm})$ & $\Omega\left(\mathrm{rad} \mathrm{s}^{-1}\right)$ & $\varepsilon\left(10^{4} \mathrm{~cm}^{2} \mathrm{~s}^{-3}\right)$ & $L_{R h}(\mathrm{~cm})$ \\
\hline $1 S$ & 2 & 4 & 3 & 2.1 & 0.6 \\
$2_{S}$ & 4 & 4 & 3 & 11.0 & 1.1 \\
$3_{S}$ & 6 & 4 & 3 & 29.0 & 1.5 \\
\hline \hline
\end{tabular}



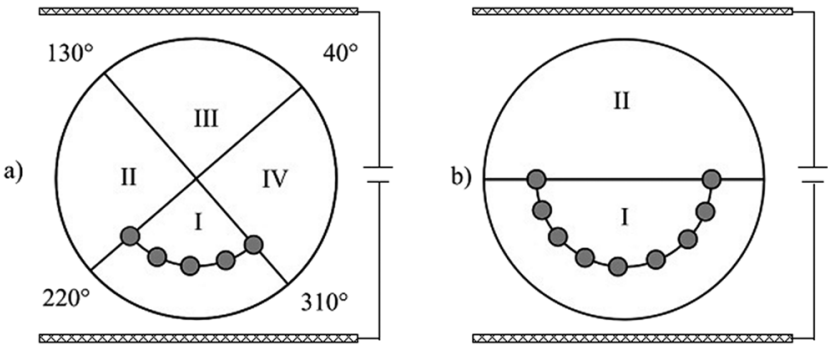

FIG. 3. Sketch of the forcing system and of forced and unforced sectors: (a) $90^{\circ}$ arc experiments and (b) $180^{\circ}$ arc experiments.

where $\varepsilon$ is the energy transfer rate toward large scale features (i.e., jets and eddies) and $k$ is the spatial wavenumber. We list the value of $\varepsilon$ for each experiment in Table I, showing that it depends on the intensity of the electric current $I$ only in both the $90^{\circ}$ and $180^{\circ}$ configurations.

\section{METHODS OF ANALYSIS}

\section{A. Characterizing waves}

The experimental configuration is such that the large-scale eddies can only be created through strong vacillations of the jet flow. If the eddies are related to Rossby waves, their phase speed would inevitably be affected by strong variability of the zonal flow. It is thus important to derive an expression for the phase speed of the Rossby waves with a mean flow $U$ that evolves on the radial direction only in the polar coordinate system. To take this into account, we introduce the effective $\beta$,

$$
\beta_{e f f} \equiv \beta+\frac{d}{d r}\left(\frac{d U}{d r}+\frac{U}{r}\right),
$$

where the parameter $\beta$ is the topographic $\beta$-effect defined in expression (2). If $\beta$ is the dominant term in (3), as it is negative in polar coordinates, then Rossby waves propagate westward.

We show in the Appendix that for linear Rossby waves in an arbitrary zonal mean flow $U(r)$ that depends on the radial direction only, the dispersion relation in polar coordinates is

$$
\omega=\frac{U(r)}{r} m+\frac{\beta_{e f f} m}{r} \frac{R^{2}}{a^{2}},
$$

where $m$ is the non-dimensional zonal mode of a wave whose phase speed is given by

$$
\frac{\omega r}{m}=U(r)+\beta_{e f f} \frac{R^{2}}{a^{2}} .
$$

In our experiments, $a, R$, and $m$ are determined by the geometry of the experimental device. Indeed, the Bessel-Fourier
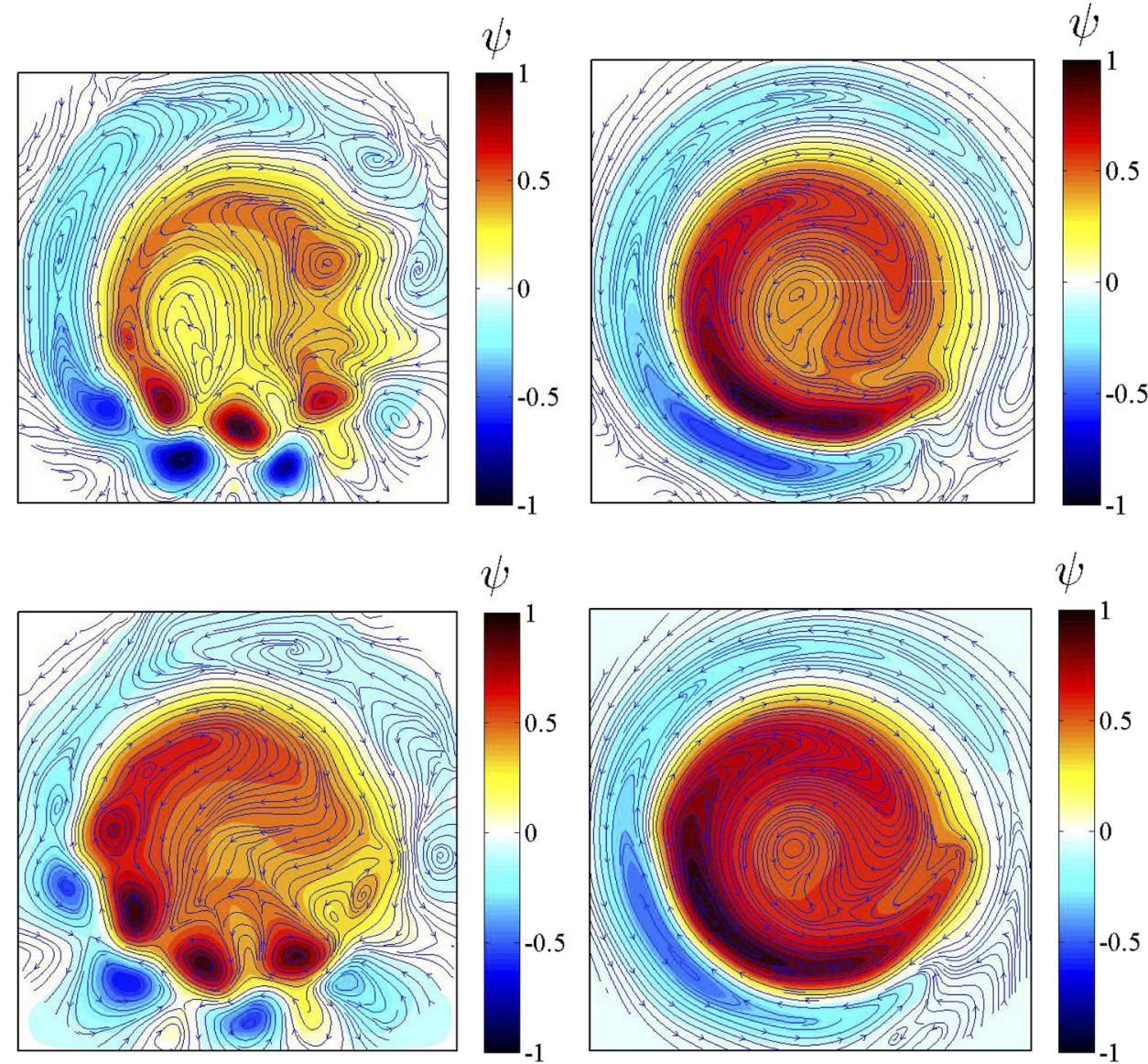

$\psi$

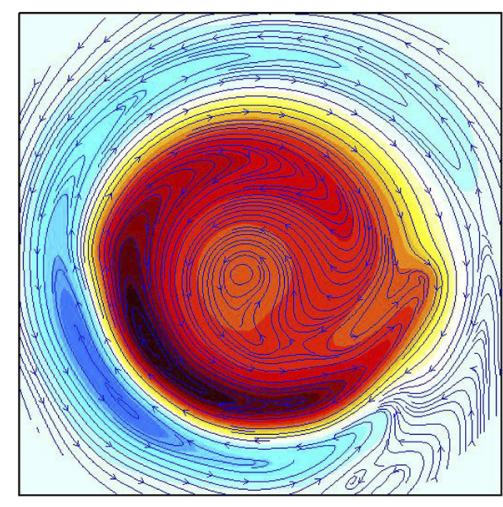

FIG. 4. Instantaneous (left) and timemean (right) streamfunction amplitudes normalized by their maximum values superimposed on the streamlines: (top) Expt. 390 and (bottom) Expt. $3_{180}$. The direction of flow is indicated by the arrows superimposed on the streamlines. The closed contours appear to identify eddy boundaries. 
decomposition establishes that $R$ is the radius of the device and $a=\alpha_{m n}{ }^{17}$ The identification of the waves and their associated zonal modes, $m$, can be computed by a spectral decomposition of the velocity fields. In the cylindrical geometry of our experimental device, the spectral decomposition of a $2 \mathrm{D}$ velocity field, $v(r, \varphi)$, is computed using the truncated Bessel-Fourier decomposition for the $90^{\circ}$ and $180^{\circ}$ sectors with the truncation index $\gamma=4$ and 2 , respectively,

$$
v(r, \varphi)=\sum_{m=-M}^{M} \sum_{n=1}^{N} C_{m}^{n} J_{m}\left(\alpha_{m n} \frac{r}{R}\right) e^{\gamma i m \varphi},
$$

where $J_{m}$ is the Bessel function of the $m$ th order, $\alpha_{m n}$ is its $n$th zero, $n$ correspond to radial modes, and $C_{m}^{n}$ are complex Bessel-Fourier coefficients. Here, $v(r, \varphi)$ is the total velocity field on the free surface of the experiment and $v(R, \varphi)=0$. With a spatial resolution of $1^{\circ}$, the total number of zonal modes $M$ is limited to 45 for a $90^{\circ}$ sector, 90 for a $180^{\circ}$ sector, and the total number of radial modes $N$ is limited to 60 (see Refs. 17 and 34). First, considering the spectral decomposition of the velocity field, one can recompute the dispersion relation expression (5) and the phase speed, with $a=\alpha_{m n}$ and $\gamma=4$ in a $90^{\circ}$ sector,

$\omega=U \frac{4 m}{r}+\beta_{\text {eff }} \frac{4 m R^{2}}{r \alpha_{4 m, n}^{2}}, \quad c_{p}=\frac{\omega r}{4 m}=U+\beta_{\text {eff }} \frac{R^{2}}{\alpha_{4 m, n}^{2}}, \quad m=1,2,3, \ldots$, and with $\gamma=2$ in a $180^{\circ}$ sector,

$$
\omega=U \frac{2 m}{r}+\beta_{\text {eff }} \frac{2 m R^{2}}{r \alpha_{2 m, n}^{2}}, \quad c_{p}=\frac{\omega r}{2 m}=U+\beta_{\text {eff }} \frac{R^{2}}{\alpha_{2 m, n}^{2}}, \quad m=1,2,3, \ldots
$$

Second, it is clear from expression (6) that one can compute the total kinetic energy $E=1 / 2 v^{2}$, leading to $2 \mathrm{D}$ energy spectra for each pair of modes $m$ and $n$, using the expression

$$
E\left(\alpha_{m n}, m\right)=\frac{1}{2} C_{m}^{n} \overline{C_{m}^{n}} J_{m+1}\left(\alpha_{m n}\right)^{2}
$$

where the over-lined coefficients are complex conjugates. In this expression, the axisymmetric (i.e., invariant around the rotational spin axis) part of the flow, namely, the jets, corresponds to $E\left(\alpha_{0 n}\right.$, $m=0)$. Waves are the non-axisymmetric parts of the flow with zonal modes $m=1,2,3, \ldots, M$. We show in Fig. 5 the energy spectra for the zonal modes $m=0, \ldots, 10$ as a function of the radial modes $n$. This figure shows that the axisymmetric $(m=0)$ flow dominates the dynamics with an energetic maximum near the typical radial scales (i.e., $4<n<7$ ) of the jet. Scale separation between the electromagnetic forcing ( $n \geq 24$ for magnets of $1 \mathrm{~cm}$ diameter) and the equilibrated jets $(4<n<7)$ argues for prevalent upscale energy transfers (see colored area in Fig. 5). At jets' typical scales in radius, both experiments depict a strong $m=1$ wave that appears to be the most energetic one at these scales. Then, the wave $m=1$ is selected in this study to be the dominant wave produced by

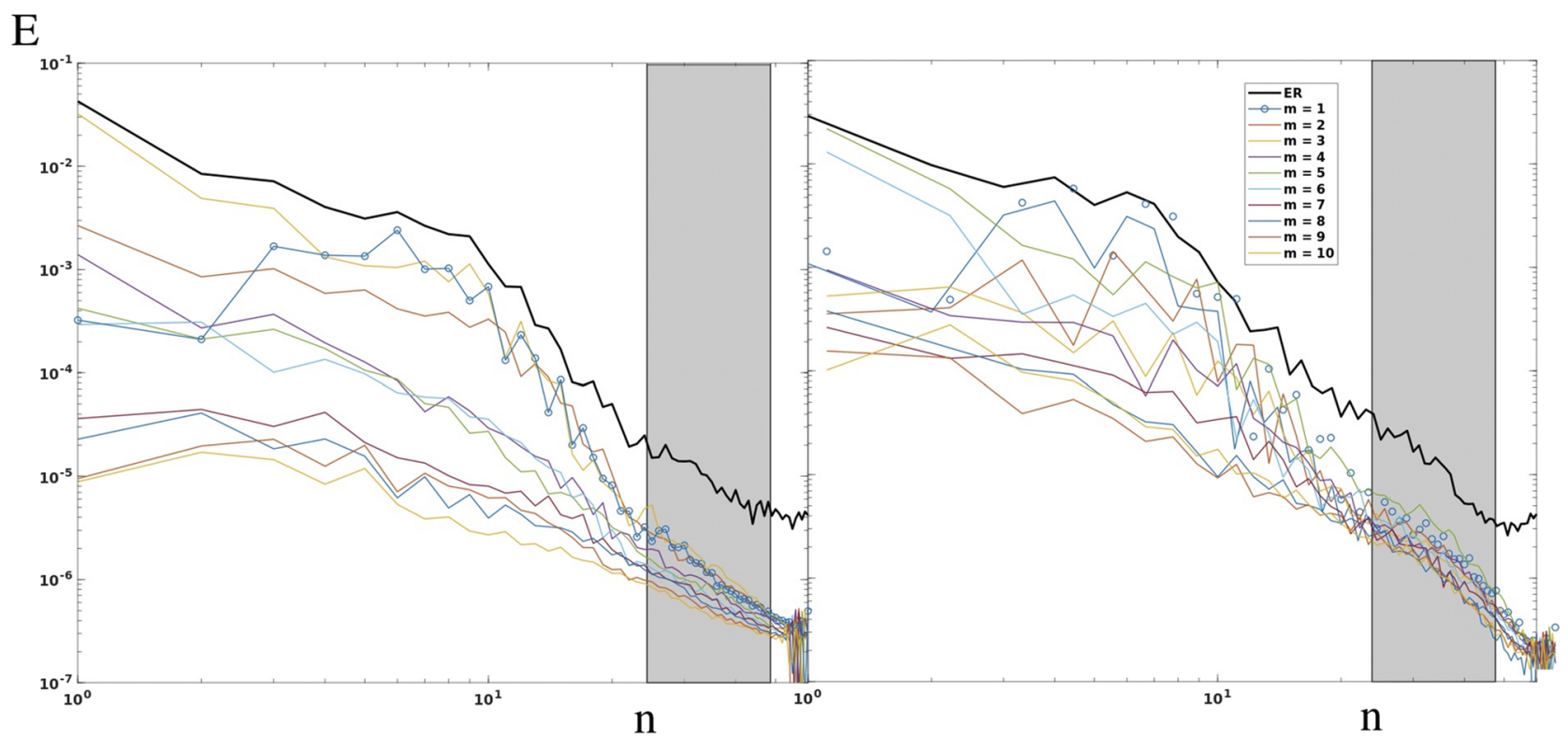

FIG. 5. Time-averaged 2D kinetic energy spectra in $\mathrm{cm}^{2} \mathrm{~s}^{-2}$ : Here, experiments (left) Expt. $3_{90}$ and (right) Expt. $3_{180}$ are represented. According to the legend, each colored spectrum corresponds to a zonal mode $m$ shown as a function of the radial modes $n$. Gray areas correspond to the injection scales, typical of the size of the magnets (1.2 $\mathrm{cm}$ corresponding to $n=48)$ and twice that size $(2.4 \mathrm{~cm}$ corresponding to $n=24)$, when including the spacing between two magnets. Brown areas correspond to radial typical scales of the jets $\approx 4 \mathrm{~cm}-7 \mathrm{~cm}$ (i.e., $n=7-4$ ), measured from the averaged zonal velocity profiles. 
a)

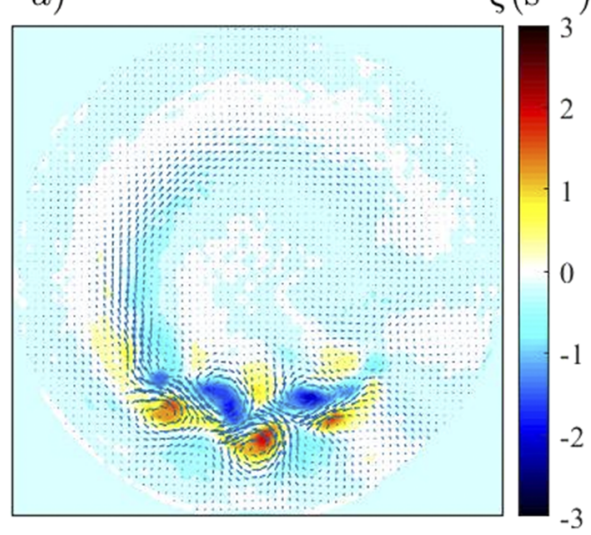

b)



FIG. 6. (a) Instantaneous fields of vorticity $(\zeta)$ and (b) Okubo-Weiss parameter $\left(Q_{o w}\right)$, with velocity vectors superimposed (Expt. $\left.3_{90}\right)$. In the $\zeta$ field, regions of dark blue (dark red) identify patches of strong anti-cyclonic (cyclonic) vorticity. In the $Q_{0 w}$ field, dark blue identifies regions where vorticity is much stronger than strain (i.e., eddy cores), and dark red where strain is much larger than vorticity (i.e., saddle point neighborhoods). the non-linear dynamics and from which the Rossby wave dispersion relation and phase speed using expressions (7) and (8) will be calculated.

\section{B. Streamfunction critical points and the Okubo-Weiss parameter}

The Okubo-Weiss parameter is defined by ${ }^{35,36}$

$$
Q_{o w}=s_{n}^{2}+s_{s}^{2}-\zeta^{2},
$$

where $s_{n}=\partial u / \partial x-\partial v / \partial y$ (normal strain), $s_{s}=\partial v / \partial x+\partial u / \partial y$ (shear strain), and $\zeta=\partial v / \partial x-\partial u / \partial y$ (vorticity). Qow allows easy identification of vorticity dominated regions (occupied by eddies) and strain dominated regions (between the eddies), as illustrated in Fig. 6(b). In order to identify eddy polarity, the vorticity field is also needed [Fig. 6(a)]. Now, recall Fig. 4 (left panels)-there $\psi$ reveals both eddy strength and polarity; however, regions of high strain are not obvious (except to the trained eye). The connection between the $\psi$ and $\left(Q_{o w}, \zeta\right)$ fields can be efficiently explained using critical point theory. Critical points are important because they, together with nearby points, influence the flow over large distances. A critical point (or singularity) $(a, b)$ of $\psi$ is where $\nabla \psi=0$. The local curvature of $\psi$ at $(a, b)$ can be determined by calculating the eigenvalues of the Hessian (the matrix of second partial derivatives) of $\psi$. From the eigenvalues, it can be determined if $(a, b)$ is a maximum, minimum, or saddle point. A simple calculation shows that the eigenvalues of the Hessian can be expressed in terms of $\zeta$ and $Q_{o w}$,

$$
\lambda_{1,2}=\frac{\zeta \pm \sqrt{\zeta^{2}+Q_{o w}}}{2}
$$

so that

(i) if vorticity is negative and dominates strain $\left(\zeta<0\right.$, $\left.Q_{o w}<0\right)$, then $\psi(a, b)$ is a local maximum (the center of an anticyclonic eddy);

(ii) if vorticity is positive and dominates strain $\left(\zeta>0, Q_{o w}<0\right)$, then $\psi(a, b)$ is a local minimum (the center of a cyclonic eddy); and

(iii) if strain dominates vorticity $\left(Q_{o w}>0\right)$, then $\psi(a, b)$ is a saddle point [their locations in the $\psi$ field (Fig. 4) are not obvious].
The next step is to identify neighborhoods within which there is (ideally) an isolated critical point. The neighborhood boundary is defined using a threshold. Here, a threshold is chosen based on the statistical distribution of $Q_{o w}$. Before doing that, however, it is instructive to consider a collection of sample probability distributions obtained for vorticity, strain, and divergence (Fig. 7).

The distribution of divergence, $\delta=\partial u / \partial x+\partial v / \partial y$, is close to Gaussian, while those for $s_{n}, s_{s}$, and $\zeta$ have fat tails. Their widths (standard deviations) are $\left(\sigma_{s_{n}}, \sigma_{s_{s}}, \sigma_{\zeta}\right)=(4.5,5.0,6.7) \sigma_{\delta}$. The Gaussian shape and small width of the $\delta$ distribution imply that divergence is small and lack strong gradients, consistent with expectations for a quasi-two-dimensional flow with weak three-dimensional circulation. The fat tails of the $\zeta, s_{n}$, and $s_{s}$ distributions show that these quantities have many extreme events. Below, it will be shown that these extreme events are localized in space.



FIG. 7. Probability distributions of vorticity $(\zeta)$, normal strain $\left(s_{n}\right)$, shear strain $\left(s_{s}\right)$, and divergence $(\delta)$ for one field taken from the experiment $3_{90}$. 

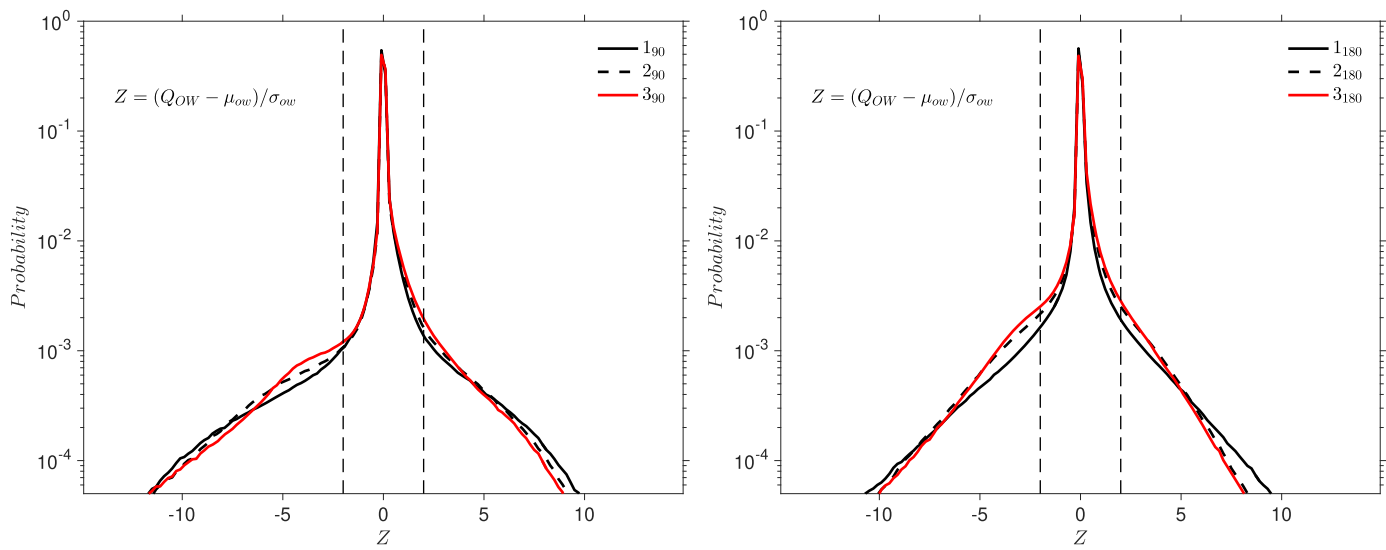

FIG. 8. Probability distribution functions calculated for each experiment (a) $90^{\circ}$ and (b) $180^{\circ}$ forcing. For ease of comparison, the distributions are plotted using standardized coordinates. Vertical dashed lines are drawn at the thresholds used to partition the data $\left( \pm Q_{c}=2 \sigma_{o w}\right)$.

Now, consider the $Q_{o w}$ probability distributions in Fig. 8 for the $90^{\circ}$ [panel (a)] and $180^{\circ}$ experiments [panel (b)]. The distributions are built using only data from the forced sector, and each is presented after transforming to standardized coordinates $\left[z=\left(Q_{o w}-\mu_{o w}\right) / \sigma_{o w}\right.$, where $\mu_{o w}$ and $\sigma_{o w}$ are, respectively, the mean and standard deviation of $\left.Q_{o w}\right]$. The distributions are approximately symmetric and have fat tails to the left and right, which are well approximated as beginning at $\pm 2 \sigma_{\text {ow }}$ (vertical dashed lines). By comparison, this is far different from the threshold often used to identify eddy boundaries $\left(0.2 \sigma_{\text {ow }}\right.$; cf. Refs. 37 and 38$)$, but consistent with that of Isern-Fontanet et al. ${ }^{39}$ who used $2 \sigma_{o w}$ to identify eddy cores in satellite altimetry data. Therefore, it seems well justified to use the threshold $Q_{c}=2 \sigma_{o w}$ to identify vorticity-dominated extremes $\left(Q_{o w}\right.$ $\left.<-Q_{c}\right)$ and strain-dominated extremes $\left(Q_{o w}>Q_{c}\right)$.

Next, a clustering algorithm is applied to the extremes of each type. The algorithm defines a cluster as a set of 10 or more contiguous pixels (two pixels are considered contiguous if they share a side or corner). If each point in the cluster satisfies $Q_{o w}<-Q_{c}$, then that cluster is classified as an $e d d y$ core. Similarly, if each point satisfies $Q_{o w}>Q_{c}$, then it is classified as a saddle point neighborhood. Eddy cores were further classified as cyclonic or anti-cyclonic, while saddle point neighborhoods were classified based on the radial location. More specifically,

(i) cyclonic eddy core (CE): $Q_{o w}<-Q_{c}$ and $\zeta>0$;

(ii) anticyclonic eddy core (AE): $Q_{o w}<-Q_{c}$ and $\zeta<0$;

(iii) Saddle Point neighborhood (SP1): $Q_{o w}>Q_{c}$ and $r<r_{C L}$; and

(iv) Saddle Point neighborhood (SP2): $Q_{o w}>Q_{c}$ and $r>r_{C L}$;

where $r=r_{C L}$ is the centerline of the mean zonal jet. The properties of each cluster, such as mean vorticity or strain, centroid location, and area, are also determined and recorded for later analysis.

Before concluding this section, it is important to note that a $2 \sigma$ threshold value is dataset-dependent. That is, the standard deviation of the data varies with spatial resolution, data quality, whether it is based on the entire domain or a subdomain, experimental parameters, etc. Thus, a $2 \sigma$ threshold could give a value of $Q_{c}$ that is smaller or larger than optimal. If it is smaller than optimal, then larger clusters will be found, and some of these could include more than one critical point. Similarly, if the $Q_{c}$ is larger than optimal, then smaller clusters will be found, and some critical points may be missed because the clusters are now too small. A judgment on the choice of $Q_{c}$ used must be considered in the analysis stage of an investigation.

\section{RESULTS}

\section{A. Propagation velocity of the Rossby waves}

The experiments produce a meandering jet and a train of eddies on either side. A diagram frequently used in meteorology and oceanography to highlight wave propagation in physical space is the Hovmöller diagram. Figure 9 shows the azimuthal ( $\varphi$-time) Hovmöller diagrams for experiments $3_{90}$ (top) and $3_{180}$ (bottom). The vertical axis is time, the horizontal axis is the angle $\varphi$, and the mapped variable is the streamfunction $\psi$ as a function of $\varphi$ at $r=r_{M 2}$ (the radius where the radial shear is a maximum, as described below). The diagrams show linear features with a negative slope, indicating westward propagating structures in the forced sectors $\left(90^{\circ}\right.$ and $180^{\circ}$, respectively). The propagating structures could be waves or eddies or both.

The slope of the contour lines in the azimuthal Hovmöller diagrams, denoted by $\Delta \varphi / \Delta t$, is easily estimated and used to calculate the propagation velocity,

$$
V_{s}=r \frac{\Delta \varphi}{\Delta t}
$$

For all experiments, the net speed of the propagating structures was evaluated by subtracting the mean zonal velocity from $V_{s}$,

$$
c=V_{s}-\left\langle\overline{V_{Z}}\right\rangle_{\varphi},
$$

where $\left\langle\overline{V_{Z}}\right\rangle_{\varphi}$ is the mean zonal velocity averaged over $\varphi$ (a sector of $90^{\circ}$ ) and time (a time interval of $\sim 200 \mathrm{~s}$ ). 

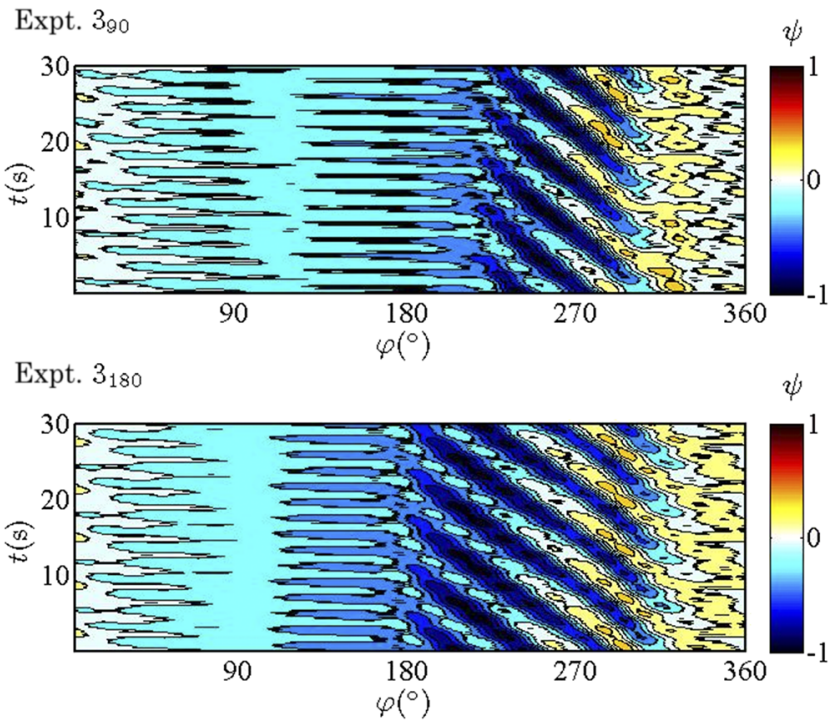

FIG. 9. Azimuthal Hovmöller diagram of the streamfunction $\psi$ at the radius of maximum radial shear $\left(r_{M 2}\right)$ : (top) Expt. $3_{90}$ and (bottom) Expt. $3_{180}$.
Figure 10 shows a tableau of results for the three $90^{\circ}$ experiments. From left-to-right, they are (a) azimuthal $\varphi$-time and (b) radial (time- $r$ ) Hovmöller diagrams, and radial profiles of (c) mean radial shear $\left(d\left\langle\overline{V_{Z}}\right\rangle_{\varphi} / d r\right)$, (d) mean azimuthal velocity $\left(\left\langle\overline{V_{Z}}\right\rangle_{\varphi}\right)$, and (e) mean potential vorticity $\left(\langle\bar{q}\rangle_{\varphi}\right)$.

We first describe the radial profiles from right-to-left and then the Hovmöller diagrams. The step-like profile shown by $\langle\bar{q}\rangle_{\varphi}$ is caused by PV mixing; this is expected as it is known that westward jets lie in zones of constant PV. The jet-profile shown by $\left\langle\overline{V_{Z}}\right\rangle_{\varphi}$ has its maximum westward speed at $r_{C L}$ (the jet centerline). The profile of the mean shear $d\left\langle\overline{V_{Z}}\right\rangle_{\varphi} / d r$ has a minimum at $r_{M 1}<r_{C L}$ and a maximum at $r_{M 2}>r_{C L}$.

We now describe the Hovmöller diagrams from left-to-right. The contours in the azimuthal Hovmöller diagrams slant up and to the left, indicating a westward propagating wave. In the radial Hovmöller diagrams, the vertical axis is $r$ and the mapped variable is $\langle\psi(r, t)\rangle_{\varphi}$ (averaged over the forced sector). The contours indicate a radial oscillation but no radial propagation.

Figure 11 shows an enlargement of the 290 radial Hovmöller diagram. Three distinctive regions can be identified: (i) a dark red region above the upper dashed line $\left(r<r_{M 1}\right)$ showing the passage of anticyclonic eddies, (ii) a dark blue region below the lower dashed line $\left(r>r_{M 2}\right)$ showing the passage of cyclonic eddies, and
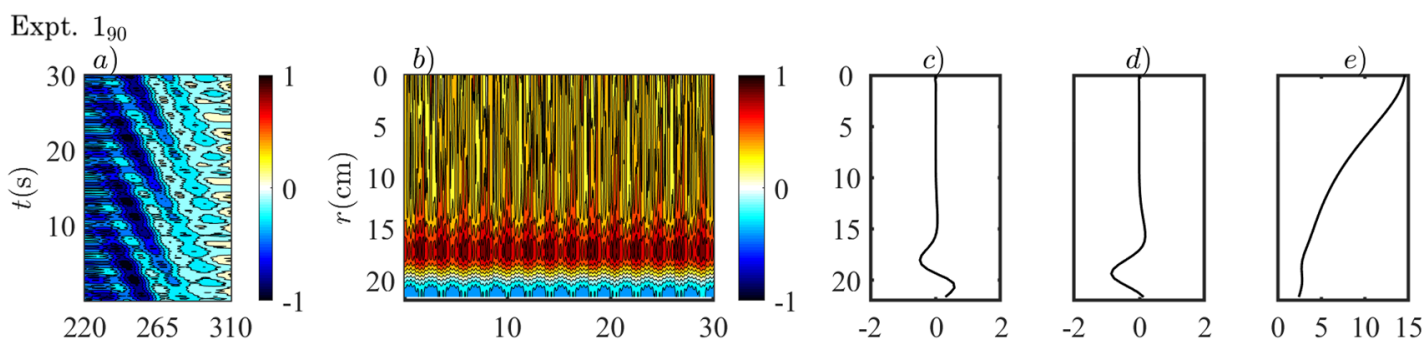

Expt. 290


FIG. 10. A tableau of results for the three $90^{\circ}$ experiments, from top to bottom: (a) Azimuthal ( $\varphi$-time) Hovmöller diagrams of the streamfunction $\psi$ at the radius of maximum radial shear $\left(r_{M 2}\right)$. (b) Radial $\left(r\right.$-time) Hovmöller diagrams of $\langle\psi(r, t)\rangle_{\varphi}$ averaged over angles $221^{\circ}-310^{\circ}$. Radial profiles of the time and $\varphi$-averaged $(c)$ radial shear $d\left\langle\overline{V_{Z}}\right\rangle_{\varphi} / d r,(d)$ azimuthal velocity $\left\langle\overline{V_{Z}}\right\rangle_{\varphi}$, and $(e)$ potential vorticity $\langle\bar{q}\rangle_{\varphi}$. Averages are calculated over angles $221^{\circ}-310^{\circ}$ and over a time interval of 4000 frames, corresponding to $200 \mathrm{~s}$. 


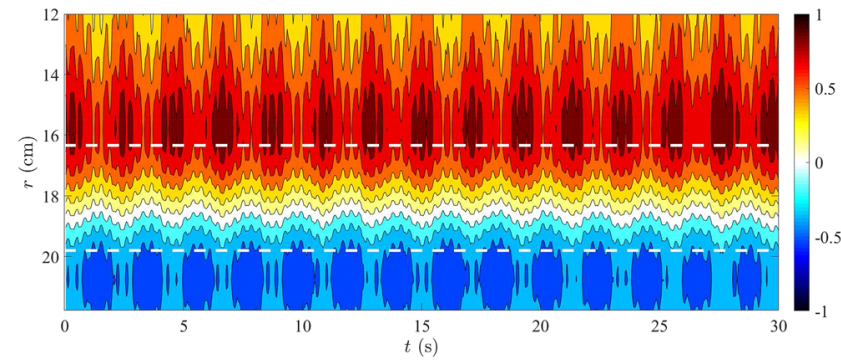

FIG. 11. Magnification of the jet region in the radial Hovmöller diagram for Expt. $2{ }_{90}$ in Fig. 10 (b). The white dashed lines indicate the radii of minimum $\left(r=r_{M 1}\right)$ and maximum $\left(r=r_{M 2}\right)$ radial shear.

(iii) a yellow region where the contours indicate the passage of a wave.

For all the experiments, we first evaluated the theoretical phase speed of the Rossby wave $c_{p}$ using Eqs. (7) and (8). We obtain the phase speeds $c_{p_{\beta}}$ and $c_{p_{\text {eff }}}$ that are computed using a constant value of $\beta$ [Eq. (2)] and using values of $\beta_{\text {eff }}$, which varies with $r$ [Eq. (3)]. They are calculated at the following radial locations: $r_{M 1}$ and $r_{M 2}$, corresponding to the minimum and maximum velocity shears, respectively; the radius $r_{C L}$ at which the mean zonal velocity is maximum; and two arbitrary radii $r_{1}$ and $r_{5}$. We list in Table II for experiment $3_{90}$ and in Table III for experiment $3_{180}$, the different radii $(r)$, the mean zonal velocity $\left[\left\langle\overline{V_{Z}}\right\rangle_{\varphi}(r)\right]$, the propagation velocity $\left(V_{s}\right)$ [Eq. (12)], the associated $\beta_{\text {eff }}$, the net speed of the propagating structures $c$ [Eq. (13)], and the propagation velocity of the Rossby waves $c_{p_{\beta_{e f f}}}$. For all experiments, the best prediction of $c$, namely, the propagation speed measured in the experiment, is obtained with the theoretical phase speed $c_{p_{\beta_{\text {eff }}}}$ at the radius of maximum shear, $r_{M 2}$. We underline that the good agreement shown in Table IV at $r_{M 1}$ is not typical across all experiments. Indeed, the relative error $\left(c / c_{p_{\beta_{\text {eff }}}}\right)$ at that location ranges between 0.09 and 0.33 with an average of 0.23 and standard deviation of 0.09 . Conversely, those at $r_{M 2}$ range between 0.01 and 0.18 with an average of 0.08 and a standard deviation of 0.07 .

Finally, we compare the net propagation speed $c$ with their theoretical predictions $c_{p_{\beta}}$ and $c_{p_{\beta_{e f f}}}$. In Tables IV and V, this comparison is computed for all experiments, at the radius of maximum agreement $r_{M 2}$. It shows that using our estimate of $\beta_{\text {eff }}$ instead of $\beta$, we significantly improve our predictions of the net propagation speed $c$. Considering this result, we claim that to estimate the phase velocity of linear Rossby waves in a jet-dominated flow, one has to consider the modified dispersion relation given in expression (4), which depends on both the radial coordinate and the mean velocity profile.

\section{B. Propagation velocity of eddies and saddle points}

Figure 12 shows the result of applying the Okubo-Weiss clustering and classification procedure (Sec. III B) to one velocity field

TABLE II. Summary of the experimental results recovered at different radii for Expt. $3_{90}: r_{M 1}\left(r_{M 2}\right)$ is the radius where the radial shear is minimum (maximum); $r_{C L}$ is the jet centerline (i.e., the radius where the mean zonal velocity is a maximum); and $r_{1}$ and $r_{5}$ are arbitrary.

\begin{tabular}{lccccccc}
\hline \hline & & & & & & \\
& $r(\mathrm{~cm})$ & $\left(\mathrm{cm} \mathrm{s}_{\ell}\right\rangle_{\varphi}(r)$ & $V_{s}\left(\mathrm{~cm} \mathrm{~s}^{-1}\right)$ & $\beta_{\text {eff }}\left(\mathrm{cm}^{-1} \mathrm{~s}^{-1}\right)$ & $c\left(\mathrm{~cm} \mathrm{~s}^{-1}\right)$ & $c_{p_{\beta_{\text {eff }}}}\left(\mathrm{cm} \mathrm{s}^{-1}\right)$ & $\mathrm{e}\left(\mathrm{c} / c_{p_{\beta_{\text {eff }}}}\right)$ \\
\hline$r_{1}$ & 14.30 & 0.37 & -1.42 & -0.95 & -1.79 & -1.48 & 0.09 \\
$r_{M 1}$ & 16.34 & -0.63 & -1.54 & -0.70 & -0.91 & -1.10 & 0.09 \\
$r_{C L}$ & 18.32 & -1.55 & -1.65 & 0.29 & -0.10 & 0.46 & 1.00 \\
$r_{M 2}$ & 19.80 & -0.69 & -1.68 & -0.55 & -0.99 & -0.86 & 0.07 \\
$r_{5}$ & 21.30 & 0.28 & -1.85 & -0.93 & -2.13 & -1.45 & 0.19 \\
\hline \hline
\end{tabular}

TABLE III. Summary of the experimental results recovered at different radii for Expt. $3_{180}: r_{M 1}$ and $r_{M 2}$ correspond to the minimum and maximum shear; $r_{C L}$ is the radius where the mean zonal velocity is maximum; and $r_{1}$ and $r_{5}$ are arbitrary.

\begin{tabular}{lccccccc}
\hline \hline & & $\left\langle\overline{V_{Z}}\right\rangle_{\varphi}(r)$ & & & & \\
& $r(\mathrm{~cm})$ & $\left(\mathrm{cm} \mathrm{s}^{-1}\right)$ & $V_{s}\left(\mathrm{~cm} \mathrm{~s}^{-1}\right)$ & $\beta_{\text {eff }}\left(\mathrm{cm}^{-1} \mathrm{~s}^{-1}\right)$ & $c\left(\mathrm{~cm} \mathrm{~s}^{-1}\right)$ & $c_{p_{\beta_{e f f}}}\left(\mathrm{~cm} \mathrm{~s}^{-1}\right)$ & $\mathrm{e}\left(\mathrm{c} / c_{p_{\beta_{e f f}}}\right)$ \\
\hline$r_{1}$ & 14.30 & 0.24 & -1.62 & -0.84 & -1.86 & -1.70 & 0.05 \\
$r_{M 1}$ & 15.97 & -0.61 & -1.56 & -0.74 & -0.96 & -1.49 & 0.22 \\
$r_{C L}$ & 17.91 & -1.59 & -1.89 & 0.24 & -0.30 & 0.49 & 1.00 \\
$r_{M 2}$ & 19.36 & -0.95 & -1.88 & -0.45 & -0.92 & -0.90 & 0.01 \\
$r_{5}$ & 21.30 & -0.05 & -2.09 & -0.81 & -2.05 & -1.63 & 0.11 \\
\hline \hline
\end{tabular}


TABLE IV. Summary of the experimental results evaluated at the radius of maximum radial shear $r_{M 2}$ for the $90^{\circ}$ forcing.

\begin{tabular}{|c|c|c|c|c|c|c|c|c|c|}
\hline Expt. & $r_{M 2}(\mathrm{~cm})$ & $\left\langle\overline{V_{Z}}\right\rangle_{\varphi}\left(\mathrm{cm} \mathrm{s}^{-1}\right)$ & $V_{s}\left(\mathrm{~cm} \mathrm{~s}^{-1}\right)$ & $\beta_{e f f}\left(\mathrm{~cm}^{-1} \mathrm{~s}^{-1}\right)$ & $c\left(\mathrm{~cm} \mathrm{~s}^{-1}\right)$ & $c_{p_{\beta_{e f f}}}\left(\mathrm{~cm} \mathrm{~s}^{-1}\right)$ & $c_{p_{\beta}}\left(\mathrm{cm} \mathrm{s}^{-1}\right)$ & $\mathrm{e}\left(\mathrm{c} / c_{p_{\beta_{e f f}}}\right)$ & $\mathrm{e}\left(\mathrm{c} / c_{p_{\beta}}\right)$ \\
\hline $1_{90}$ & 20.75 & -0.25 & -1.05 & -0.59 & -0.80 & -0.92 & -0.83 & 0.07 & 0.02 \\
\hline 290 & 19.80 & -0.46 & -1.38 & -0.57 & -0.92 & -0.89 & -0.83 & 0.02 & 0.05 \\
\hline $3_{90}$ & 19.80 & -0.69 & -1.68 & -0.55 & -0.99 & -0.86 & -0.83 & 0.07 & 0.09 \\
\hline
\end{tabular}

TABLE V. Summary of the experimental results evaluated at the radius of maximum radial shear $r_{M 2}$ for the $180^{\circ}$ forcing.

\begin{tabular}{|c|c|c|c|c|c|c|c|c|c|}
\hline Expt. & $r_{M 2}(\mathrm{~cm})$ & $\left\langle\overline{V_{Z}}\right\rangle_{\varphi}\left(\mathrm{cm} \mathrm{s}^{-1}\right)$ & $V_{s}\left(\mathrm{~cm} \mathrm{~s}^{-1}\right)$ & $\beta_{e f f}\left(\mathrm{~cm}^{-1} \mathrm{~s}^{-1}\right)$ & $c\left(\mathrm{~cm} \mathrm{~s}^{-1}\right)$ & $c_{p_{\beta_{e f f}}}\left(\mathrm{~cm} \mathrm{~s}^{-1}\right)$ & $c_{p_{\beta}}\left(\mathrm{cm} \mathrm{s}^{-1}\right)$ & $\mathrm{e}\left(\mathrm{c} / c_{p_{\beta_{e f f}}}\right)$ & $\mathrm{e}\left(\mathrm{c} / c_{p_{\beta}}\right)$ \\
\hline $1_{180}$ & 19.36 & -0.31 & -1.25 & -0.51 & -0.94 & -1.03 & -1.07 & 0.04 & 0.06 \\
\hline $22_{180}$ & 19.36 & -0.67 & -1.61 & -0.45 & -0.94 & -0.91 & -1.07 & 0.02 & 0.06 \\
\hline $3_{180}$ & 19.36 & -0.95 & -1.88 & -0.45 & -0.92 & -0.90 & -1.07 & 0.01 & 0.07 \\
\hline
\end{tabular}

taken from experiment 290 . Clusters were identified using the threshold $Q_{c}=2 \sigma_{o w}$, where $\sigma_{o w}$ is the standard deviation calculated using all data in the time series in the forced sector. In this figure, the strongest and largest clusters are in the central region of the forced sector. Their centers (marked with an " $x$ ") lie close to the dashed arcs, which are drawn at radii $r_{M 1}$ and $r_{M 2}$ where the mean radial shear is a minimum and maximum, respectively. The green arc indicates the location of the magnets used to force the flow (the magnets are $1 \mathrm{~cm}$ in diameter and their centers are at $r=17 \mathrm{~cm})$. Contours are drawn at $-0.2 \sigma_{o w},-2 \sigma_{o w}$, and $2 \sigma_{o w}$.

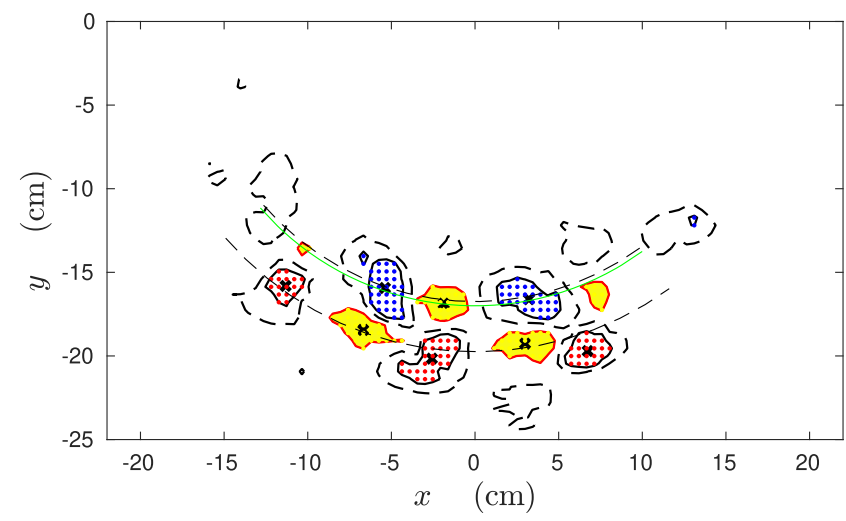

FIG. 12. A snapshot of an eddy field from Expt. $2{ }_{90}$. The $-0.2 \sigma_{0 w}$ contour (dashed) represents the outer boundary of an eddy; strong eddies have an inner core (enclosed by the $-2 \sigma_{0 w}$ contour), while weak eddies do not. Eddy cores with red (blue) dots have cyclonic (anti-cyclonic) vorticity. Saddle point neighborhoods are defined by the $2 \sigma_{o w}$ contour and filled yellow (the $0.2 \sigma_{o w}$ contour is not shown). Those clusters with area greater than ten pixels are marked with an " $x$ " at their center. The inner and outer dashed arcs are at radii where the mean radial shear is a local minimum $\left(r_{M 1}\right)$ and a local maximum $\left(r_{M 2}\right)$. The green arc indicates the location of the magnets used to force the flow (the magnets are $1 \mathrm{~cm}$ in diameter and their centers are at $r=17 \mathrm{~cm}$ ).
The $-0.2 \sigma_{\text {ow }}$ contour (dashed) defines the outer boundary of an eddy, ${ }^{37,38}$ while eddy cores are defined by the $-2 \sigma_{\text {ow }}$ contour. The color of the dots indicates eddy polarity: blue dots indicate anticyclonic cores and red dots cyclonic cores. The neighborhood of a saddle point is enclosed by the $2 \sigma_{o w}$ contour and filled yellow. Note that in the experiments, the clusters move in the clockwise (westward) direction.

With each successive frame, a pattern of clusters advance in the clockwise direction, with new clusters created in the entrance region of the forced sector, growing to maximum size in the center, and then decreasing in size as they approach the end of the sector. A summary of this process over the course of an experiment is shown for each cluster type in Fig. 13. For example, each point in the top-left panel represents the area and angular position of every anti-cyclonic eddy in the experimental time series.

Figure 14 plots the location of all cluster centers (separated by type) found in the Expt. 290 time series. Inspection of Figs. 11 and 12 suggests that the centers of eddies and saddle points will be narrowly distributed about a mean radial location (near $r_{M 1}$ for $\mathrm{AE}$ and SP1 clusters and $r_{M 2}$ for CE and SP2 clusters). While Fig. 14 shows that this is true for the AE and SP2 clusters, it also shows that CE and SP1 clusters are broadly distributed. The reason for this is not fully understood but could be due to errors in clustering caused by experimental noise. However, we do not believe that this affects the conclusions of this paper.

The strength of the eddies can be quantified using the eddy Rossby number $R o_{\text {eddy }}=|\bar{\zeta} / f|$ (where $f=2 \Omega$ ), which measures the extent to which an eddy is influenced by cyclostrophic as well as geostrophic forces. ${ }^{40}$ In the oceanographic context, those eddies with a sufficiently large value of $R o_{e d d y}$ are called "nonlinear" and play a major role in the redistribution of heat, salinity, and nutrients. According to the analysis of Ref. $40, R o_{e d d y}>0.2$ are nonlinear eddies and $R o_{e d d y}>0.3$ are strongly nonlinear. Figure 15 shows that the eddies in our experiments are nonlinear, but not strongly so.

We now extend our analysis to characterize the propagation velocities of the eddies and saddle points in order to relate them 

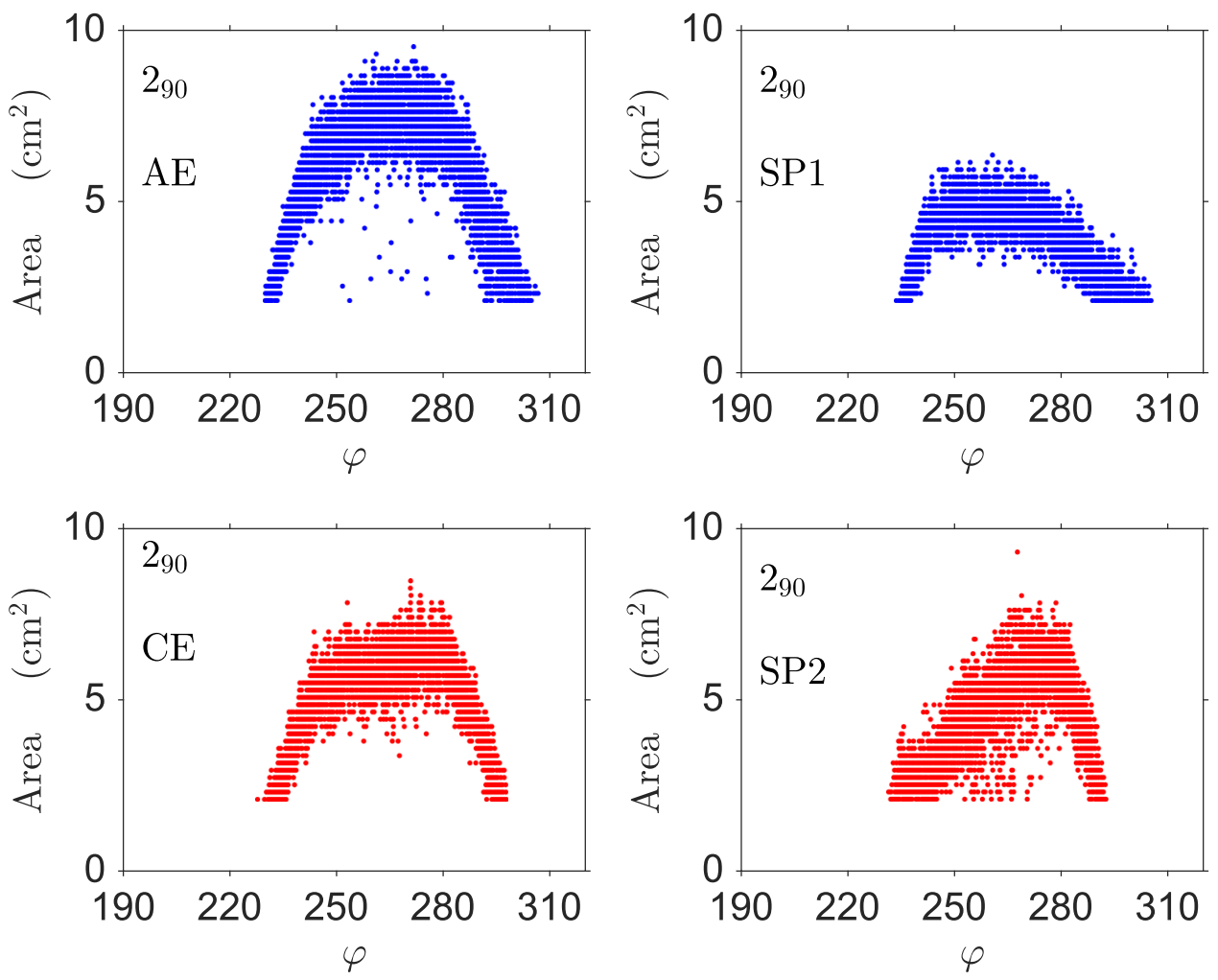

FIG. 13. Distribution of cluster size as a function of its angular position. AE: anticyclonic eddies; CE: cyclonic eddies; SP1: saddle points (inner); and SP2: saddle points (outer) (Expt. 290).

to the traveling Rossby waves. The propagation velocity of eddy cores and saddle point neighborhoods can be calculated with the position $\varphi$ against snapshot time. The advancing pattern produces aid of the azimuthal Hovmöller diagram in Fig. 16. This diagram is obtained by plotting, for each cluster type, the cluster angular the parallel linear features, and the different colors correspond to different cluster types. In the diagram, AE points fall on top of SP2 points, and similarly for CE and SP1. The slope of the linear


FIG. 14. Radial distribution of clusters as a function of their angular position. AE: anti-cyclonic eddies; $\mathrm{CE}$ : cyclonic eddies; SP1: saddle points (inner); and SP2: saddle points (outer) (Expt. $2_{90}$ ). The dashed lines are drawn at the radius of minimum and maximum radial shear $\left(r_{M 1}\right.$ and $\left.r_{M 2}\right)$ and at the radius of the jet centerline $\left(r_{C L}\right)$. 


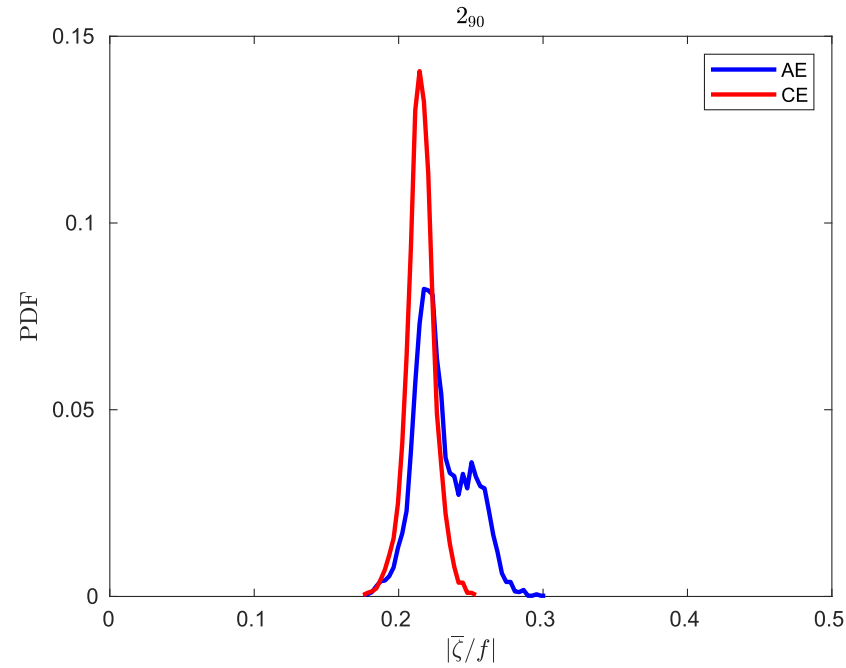

FIG. 15. PDF of the nonlinearity parameter $R o_{e d d y}=|\bar{\zeta} / f|$.

features is estimated with a least-squares fit to obtain $(\Delta \varphi / \Delta t)_{o w}$ and compared in Table VI with $(\Delta \varphi / \Delta t)_{\psi}$ obtained from the streamfunction azimuthal Hovmöller diagrams. As can be seen, they are in excellent agreement, and hence, their propagation velocities, $\left(V_{s}\right)_{o w}$ $=r_{M 2}(\Delta \varphi / \Delta t)_{o w}$, are also in excellent agreement with the theoretical propagation velocity calculated from Eq. (7) for the case of an $m=1$ Rossby wave.

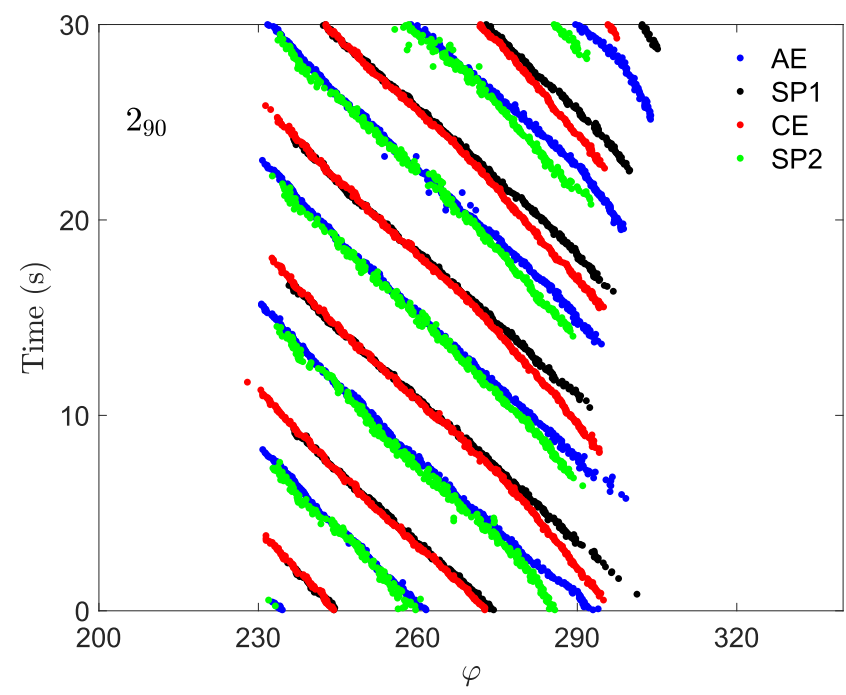

FIG. 16. Hovmöller diagrams for eddy cores and saddle point neighborhoods (Expt. 290). The figure shows, for each cluster type, the cluster angular position $\varphi$ against snapshot time. The slope of the linear features is approximately equal and denoted in the text by $(\Delta \varphi / \Delta t)_{\text {ow }}$.
TABLE VI. Comparison of $\Delta \varphi / \Delta t$ estimated from the streamfunction and OkuboWeiss analyses (units: $\operatorname{rad~s}^{-1}$ ).

\begin{tabular}{lcc}
\hline \hline Expt. & $(\Delta \varphi / \Delta t)_{\psi}$ & $(\Delta \varphi / \Delta t)_{o w}$ \\
\hline $1_{90}$ & -0.051 & -0.046 \\
$2_{90}$ & -0.072 & -0.069 \\
$3_{90}$ & -0.095 & -0.085 \\
$1_{180}$ & -0.065 & -0.057 \\
$2_{180}$ & -0.083 & -0.081 \\
$3_{180}$ & -0.097 & -0.100 \\
\hline \hline
\end{tabular}

\section{DISCUSSION AND CONCLUSIONS}

The overall goal of our work is to gain insight into the structures resulting from the complex interaction of jets, eddies, and waves through upscale/downscale turbulent cascades that are ubiquitous in the atmospheres of giant planets and in the Earth's oceans. The specific objectives of this paper were to (i) validate theoretical corrections to the phase speed arising from curvature effects, (ii) characterize the wave and eddies, and then (iii) consider the relation between the eddies and the Rossby wave-if they were independent, co-existing, or inseparable structures. To attain those objectives, a series of experiments were carried out in a rotating tank designed to excite a single westward jet in a quasi-two-dimensional barotropic rotating fluid through small-scale electromagnetic forcing confined to a range of longitudes (a $90^{\circ}$ or $180^{\circ}$ sector). A time series of twodimensional velocity fields were measured, which showed a meandering jet and a train of westward propagating eddies on both sides of the jet axis (anticyclonic to the north and cyclonic to the south). After time averaging, the mean flow appeared as a system of alternating zonal jets: a strong westward jet flanked on each side by a weak eastward jet.

The velocity fields were used to calculate the streamfunction, relative vorticity, divergence, shear and strain rate, which were, in turn, used to compute the radial profiles of the azimuthal velocity, shear, and potential vorticity. Then, for all the experiments, the slope of the contour lines in the azimuthal Hovmöller diagrams was estimated and used to calculate the propagation velocity $V_{s}$; the net speed of the propagating structures was then evaluated by subtracting the mean zonal velocity.

The experimental configuration is such that the large-scale eddies could only be created through strong vacillations of the jet flow. If the eddies are related to the Rossby waves, their phase speed would inevitably be affected by strong variability of the zonal flow; an expression for the phase speed was derived to include the radial variation of the mean zonal flow [Eqs. (3) and (4)], but it was beyond the scope of the theory to specify what value of $r$ to use to calculate the Rossby wave propagation speed. Therefore, it was necessary to estimate phase and propagation speeds from slopes of the streamfunction azimuthal Hovmöller diagrams mapped at different values of $r$.

An independent estimate of the phase speed was made from the Okubo-Weiss analysis. It showed that all eddy cores and saddle point neighborhoods, identified as clusters according to the value of the Okubo-Weiss parameter $Q_{o w}$, propagated with the same 
angular phase speed $(\Delta \varphi / \Delta t)$. The result of all these estimates demonstrated that the best match between the propagating structures in the experiments and the theoretical propagation speed was obtained at the radius of maximum shear, $r=r_{M 2}$-the position suggested by Ref. 3 .

Furthermore, we showed that the modified dispersion relation given in Eq. (4), which depends on both the radial coordinate and the mean velocity profile, significantly improved predictions of the net propagation speed. To this concern, we recall that while in Cartesian coordinates, this effect is easy to account for; in curvilinear coordinates, the impact of the curvature of the zonal velocity profile is non-trivial, and moreover, almost never included in the calculations.

In summary, the small scale forcing generates a westward meandering jet. The wavy amplitude is large enough to cause the waves to roll up into eddies on both sides of the jet. The eddy centers are located near local maxima in the magnitude of the radial shear. The distribution of eddy Rossby numbers shows that the eddies are "nonlinear" - that is, the eddies are nonlinear waves. The eddies were found to propagate at a speed close to that expected for linear Rossby waves. This indicates the likelihood of strong resonance effects and promotes the interpretation that the train of eddies is a Rossby soliton (see Ref. 41 and references therein).

\section{ACKNOWLEDGMENTS}

S.E. and G.D.N. thank the Sapienza University of Rome (Research Project No. RM11615503471374). S.C. and S.E. thank the European Union's Horizon 2020 research and innovation programme (Marie Sklodowska-Curie Grant Agreement No. 797012). G.P.K. and B.G. were partially supported by the NASA/NOAA Ocean Surface Topography Science Team (Grant Nos. 2500171500 and 2500171600) and by a University of South Florida Nexus Initiative (UNI) Award (No. RP0101).

\section{APPENDIX: DERIVATION OF THE DISPERSION RELATION OF ROSSBY WAVES IN POLAR COORDINATES AND IN A GENERAL FORM}

In this Appendix, we derive an expression for the phase speed of the Rossby waves with a mean flow that changes in the radial direction of the polar coordinate system. To this aim, one writes the governing equations neglecting the vertical velocity and denoting by tilde the instantaneous time- and space-dependent variables,

$$
\begin{aligned}
& \frac{\partial \tilde{u}}{\partial t}+(\tilde{\boldsymbol{u}} \cdot \nabla) \tilde{u}-\frac{\tilde{v}^{2}}{r}-f \tilde{v}=-\frac{1}{\rho} \frac{\partial \tilde{p}}{\partial r}, \\
& \frac{\partial \tilde{v}}{\partial t}+(\tilde{\boldsymbol{u}} \cdot \nabla) \tilde{v}+\frac{\tilde{u} \tilde{v}}{r}+f \tilde{u}=-\frac{1}{r \rho} \frac{\partial \tilde{p}}{\partial \phi},
\end{aligned}
$$

where

$$
\begin{aligned}
(\tilde{\boldsymbol{u}} \cdot \nabla) F & =\tilde{u} \frac{\partial F}{\partial r}+\frac{\tilde{v}}{r} \frac{\partial F}{\partial \phi}, \\
\tilde{\boldsymbol{u}} & =\{\tilde{u}, \tilde{v}\},
\end{aligned}
$$

with $F$ being an arbitrary function, and the continuity equation is

$$
\frac{1}{r} \frac{\partial(r \tilde{u})}{\partial r}+\frac{1}{r} \frac{\partial \tilde{v}}{\partial \phi}=0 .
$$

Introduce the decomposition $\tilde{u}=u, \tilde{v}=U(r)+v, \tilde{p}=P+p$, and rewrite the governing equations,

$$
\begin{aligned}
\frac{\partial u}{\partial t}+ & u \frac{\partial u}{\partial r}+\frac{U+v}{r} \frac{\partial u}{\partial \phi}-\frac{(U+v)^{2}}{r}-f(U+v) \\
& =-\frac{1}{\rho} \frac{\partial(P+p)}{\partial r}, \\
\frac{\partial v}{\partial t}+ & u \frac{\partial(v+U)}{\partial r}+\frac{U+v}{r} \frac{\partial(U+v)}{\partial \phi}+\frac{u(U+v)}{r}+f u \\
= & -\frac{1}{r \rho} \frac{\partial(P+p)}{\partial \phi} .
\end{aligned}
$$

Linearize these equations considering that $U(r)$ is in geostrophic balance (i.e., $\partial P / \partial r=\rho f U$ ),

$$
\begin{gathered}
\frac{\partial u}{\partial t}+\frac{U}{r} \frac{\partial u}{\partial \phi}-\frac{2 U v}{r}-f v=-\frac{1}{\rho} \frac{\partial p}{\partial r}, \\
\frac{\partial v}{\partial t}+u \frac{\partial U}{\partial r}+\frac{U}{r} \frac{\partial v}{\partial \phi}+\frac{u U}{r}+f u=-\frac{1}{r \rho} \frac{\partial p}{\partial \phi} .
\end{gathered}
$$

The continuity equation does not change.

Differentiate (A6) by $\phi$, multiply (A7) by $r$, and then differentiate by $r$, and finally, subtract the latter from the former to obtain

$$
\begin{gathered}
\frac{\partial}{\partial t}\left[\frac{\partial u}{\partial \phi}-\frac{\partial}{\partial r}(r v)\right]+\frac{U}{r} \frac{\partial^{2} u}{\partial \phi^{2}}-\frac{2 U}{r} \frac{\partial v}{\partial \phi}-U^{\prime} u-r U^{\prime} \frac{\partial u}{\partial r}-r u U^{\prime \prime} \\
-U^{\prime} \frac{\partial v}{\partial \phi}-U \frac{\partial^{2} v}{\partial r \partial \phi}-U^{\prime} u-U \frac{\partial u}{\partial r}-r u \frac{d f}{d r}=0
\end{gathered}
$$

Grouping the terms containing $U^{\prime}$ and taking the latter as a factor, find

$$
U^{\prime}\left(r \frac{\partial u}{\partial r}+u+\frac{\partial v}{\partial \phi}\right)=0
$$

by incompressibility (A4). Group the terms proportional to $U$,

$$
\begin{aligned}
\frac{U}{r} \frac{\partial^{2} u}{\partial \phi^{2}}-\frac{U}{r} \frac{\partial v}{\partial \phi}-U \frac{\partial^{2} v}{\partial r \partial \phi} & =\frac{U}{r} \frac{\partial}{\partial \phi}\left(\frac{\partial u}{\partial \phi}-r \frac{\partial v}{\partial r}-v\right) \\
& =\frac{U}{r}\left[\frac{\partial u}{\partial \phi}-\frac{\partial}{\partial r}(r v)\right],
\end{aligned}
$$

after which Eq. (A8) simplifies even more,

$$
\begin{aligned}
& \frac{\partial}{\partial t}[\left.\frac{\partial u}{\partial \phi}-\frac{\partial}{\partial r}(r v)\right]+\frac{U}{r} \frac{\partial}{\partial \phi}\left[\frac{\partial u}{\partial \phi}-\frac{\partial}{\partial r}(r v)\right] \\
&-\frac{U}{r} \frac{\partial v}{\partial \phi}-U^{\prime} u-r u U^{\prime \prime}-U \frac{\partial u}{\partial r}-r u \frac{d f}{d r}=0 .
\end{aligned}
$$

By incompressibility,

$$
\frac{U}{r} \frac{\partial v}{\partial \phi}+U \frac{\partial u}{\partial r}=\frac{U}{r}\left(r \frac{\partial u}{\partial r}+\frac{\partial v}{\partial \phi}\right)=-\frac{U u}{r},
$$


and so the first four terms in the second line in (A11) become

$$
-u r U^{\prime \prime}-u U^{\prime}+\frac{u U}{r}=-u\left(r U^{\prime \prime}+U^{\prime}-\frac{U}{r}\right) .
$$

Noting that

$$
\frac{d}{d r}\left(\frac{U}{r}\right)=\frac{U^{\prime}}{r}-\frac{U}{r^{2}}=\frac{1}{r}\left(\frac{d U}{d r}-\frac{U}{r}\right)
$$

such that

$$
U^{\prime}-\frac{U}{r}=r \frac{d}{d r}\left(\frac{U}{r}\right)
$$

we find

$$
r U^{\prime \prime}+U^{\prime}-\frac{U}{r}=r U^{\prime \prime}+r \frac{d}{d r}\left(\frac{U}{r}\right)=r \frac{d}{d r}\left(\frac{d U}{d r}+\frac{U}{r}\right) .
$$

Finally, we can write, in the linear approximation,

$$
\begin{gathered}
\frac{\partial}{\partial t}\left[\frac{\partial u}{\partial \phi}-\frac{\partial}{\partial r}(r v)\right]+\frac{U}{r} \frac{\partial}{\partial \phi}\left[\frac{\partial u}{\partial \phi}-\frac{\partial}{\partial r}(r v)\right] \\
=r u\left[\frac{d f}{d r}+\frac{d}{d r}\left(\frac{d U}{d r}+\frac{U}{r}\right)\right] \\
=r u\left[\beta+\frac{d}{d r}\left(\frac{d U}{d r}+\frac{U}{r}\right)\right] .
\end{gathered}
$$

Introduce $\beta_{\text {eff }}$ as

$$
\beta_{\text {eff }} \equiv \beta+\frac{d}{d r}\left(\frac{d U}{d r}+\frac{U}{r}\right) .
$$

Note that in polar coordinates, $d f / d r<0$ and so $\beta=d f / d r$ $<0$, while in the conventional geophysical coordinates, $\beta>0$; Rossby waves propagate westward in the polar coordinate system if $\beta$ is the dominant term in (A18).

Introduce the streamfunction $\psi$ according to

$$
\begin{aligned}
& u=\frac{1}{r} \frac{\partial \psi}{\partial \phi}, \\
& v=-\frac{\partial \psi}{\partial r}
\end{aligned}
$$

in which case

$$
\begin{aligned}
\frac{\partial u}{\partial \phi}-\frac{\partial}{\partial r}(r v) & =\frac{\partial}{\partial \phi}\left(\frac{1}{r} \frac{\partial \psi}{\partial \phi}\right)+\frac{\partial}{\partial r}\left(r \frac{\partial \psi}{\partial r}\right) \\
& =r\left(\frac{\partial^{2} \psi}{\partial r^{2}}+\frac{1}{r} \frac{\partial \psi}{\partial r}+\frac{1}{r^{2}} \frac{\partial^{2} \psi}{\partial \phi^{2}}\right)=r \nabla^{2} \psi
\end{aligned}
$$

after which Eq. (A17) becomes

$$
\frac{\partial}{\partial t}\left(r \nabla^{2} \psi\right)+U \frac{\partial}{\partial \phi} \nabla^{2} \psi=\beta_{e f f} \frac{\partial \psi}{\partial \phi} .
$$

Recall that the canonical equation for a Bessel function with the index $m, J_{m}(r)$, is

$$
\frac{\partial^{2} J_{m}(r)}{\partial r^{2}}+\frac{1}{r} \frac{\partial J_{m}(r)}{\partial r}+\left(1-\frac{m^{2}}{r^{2}}\right) J_{m}(r)=0
$$

or

$$
J_{m}(r)=-\left(\frac{\partial^{2}}{\partial r^{2}}+\frac{1}{r} \frac{\partial}{\partial r}-\frac{m^{2}}{r^{2}}\right) J_{m}(r) .
$$

It is easy to see that this equation holds for any Bessel function argument of the type $\zeta=\operatorname{ar} / R$, where $a$ and $R$ are constants; in this case,

$$
J_{m}(\zeta)=-\left(\frac{a}{R}\right)^{2}\left(\frac{\partial^{2}}{\partial \zeta^{2}}+\frac{1}{\zeta} \frac{\partial}{\partial \zeta}-\frac{m^{2}}{\zeta^{2}}\right) J_{m}(\zeta)
$$

Let us now assume that the streamfunction $\psi$ can be represented as $\psi(r, \phi, t)=\exp [i(m \phi-\omega t)] J_{m}(\operatorname{ar} / R)$. Using Eqs. (A21)-(A25), one recovers the dispersion relation for the linear Rossby waves in an arbitrary zonal flow in polar coordinates,

$$
\omega=\frac{U(r)}{r} m+\frac{\beta_{e f f} m}{r} \frac{R^{2}}{a^{2}} .
$$

This is the dispersion relation of the linear Rossby waves described by the original system of equations (see Refs. 24 and 42), except that we use $\beta_{\text {eff }}$ instead of $\beta$. The phase speed of the wave $m$ is

$$
\frac{\omega r}{m}=U(r)+\beta_{\text {eff }} \frac{R^{2}}{a^{2}} .
$$

\section{DATA AVAILABILITY}

The data that support the findings of this study are openly available in Zenodo at http://doi.org/10.5281/zenodo.3634814.

\section{REFERENCES}

${ }^{1}$ M. P. Baldwin, P. B. Rhines, H.-P. Huang, and M. E. McIntyre, "The jet-stream conundrum," Science 315, 467-468 (2007).

${ }^{2}$ D. G. Dritschel and M. E. McIntyre, "Multiple jets as PV staircases: The Phillips effect and the resilience of eddy-transport barriers," J. Atmos. Sci. 65, 855-874 (2008).

${ }^{3}$ S. Sukoriansky, N. Dikovskaya, R. Grimshaw, and B. Galperin, "Rossby waves and zonons in zonostrophic turbulence," AIP Conf. Proc. 1439, 111-122 (2012).

${ }^{4}$ L. Li, A. P. Ingersoll, A. R. Vasavada, C. C. Porco, A. D. Del Genio, and S. P. Ewald, "Life cycles of spots on Jupiter from Cassini images," Icarus 172, 9-23 (2004).

${ }^{\mathbf{5}}$ J. Legarreta and A. Sánchez-Lavega, "Vertical structure of Jupiter's troposphere from nonlinear simulations of long-lived vortices," Icarus 196, 184-201 (2008).

${ }^{6}$ H. J. Trammell, L. Li, X. Jiang, M. Smith, S. Hörst, and A. Vasavada, "The global vortex analysis of Jupiter and Saturn based on Cassini imaging science subsystem," Icarus 242, 122-129 (2014)

${ }^{7}$ K. M. Sayanagi, U. A. Dyudina, S. P. Ewald, G. D. Muro, and A. P. Ingersoll, “Cassini ISS observation of Saturn's string of pearls," Icarus 229, 170-180 (2014).

${ }^{8}$ R. G. Cosentino, A. Simon, R. Morales-Juberias, and K. M. Sayanagi, “Observations and numerical modeling of the Jovian Ribbon," Astrophys. J. 810, L10 (2015).

${ }^{9}$ E. García-Melendo and A. Sánchez-Lavega, "A study of the stability of Jovian zonal winds from HST images: 1995-2000,” Icarus 152, 316-330 (2001).

${ }^{10}$ L. Qiao and R. H. Weisberg, "Tropical instability wave kinematics: Observations from the tropical instability wave experiment," J. Geophys. Res.: Oceans $\mathbf{1 0 0}$ 8677-8693, https://doi.org/10.1029/95jc00305 (1995).

${ }^{11}$ D. B. Chelton, F. J. Wentz, C. L. Gentemann, R. A. de Szoeke, and M. G. Schlax, "Satellite microwave SST observations of transequatorial tropical instability waves," Geophys. Res. Lett. 27, 1239-1242, https://doi.org/10.1029/1999gl011047 (2000).

${ }^{12}$ R. F. Contreras, "Long-term observations of tropical instability waves," J. Phys. Oceanogr. 32, 2715-2722 (2002).

${ }^{13}$ C. S. Willett, R. R. Leben, and M. F. Lavín, "Eddies and Tropical Instability Waves in the eastern tropical Pacific: A review," Prog. Oceanogr. 69, 218-238 (2006). 
${ }^{14}$ P. Dutrieux, C. E. Menkes, J. Vialard, P. Flament, and B. Blanke, "Lagrangian study of tropical instability vortices in the Atlantic," J. Phys. Oceanogr. 38, 400417 (2008).

${ }^{15} \mathrm{~J}$.-Y. Yu and W. T. Liu, "A linear relationship between ENSO intensity and tropical instability wave activity in the eastern Pacific Ocean," Geophys. Res. Lett. 30, 1735, https://doi.org/10.1029/2003gl017176 (2003).

${ }^{16}$ P. S. Polito and O. T. Sato, “Do eddies ride on Rossby waves?," J. Geophys. Res.: Oceans 120, 5417-5435, https://doi.org/10.1002/2015jc010737 (2015).

${ }^{17}$ B. Galperin, J. Hoemann, S. Espa, and G. D. Nitto, "Anisotropic turbulence and Rossby waves in an easterly jet: An experimental study," Geophys. Res. Lett. 41, 6237-6243, https://doi.org/10.1002/2014gl060767 (2014).

${ }^{18}$ B. Galperin, J. Hoemann, S. Espa, G. Di Nitto, and G. Lacorata, "Anisotropic macroturbulence and diffusion associated with a westward zonal jet: From laboratory to planetary atmospheres and oceans,” Phys. Rev. E 94, 063102 (2016).

${ }^{19}$ S. Espa, G. F. Carnevale, A. Cenedese, and M. Mariani, "Quasi-two-dimensional decaying turbulence subject to the $\beta$ effect," J. Turbul. 9, N36 (2008).

${ }^{20}$ S. Espa, A. Cenedese, M. Mariani, and G. F. Carnevale, "Quasi-two-dimensional flow on the polar $\beta$-plane: Laboratory experiments," J. Mar. Syst. 77, 502-510 (2009).

${ }^{21}$ S. Espa, G. Di Nitto, and A. Cenedese, "The emergence of zonal jets in forced rotating shallow water turbulence: A laboratory study," EPL 92, 34006 (2010).

${ }^{22}$ S. Espa, I. Bordi, T. Frisius, K. Fraedrich, A. Cenedese, and A. Sutera, "Zonal jets and cyclone-anticyclone asymmetry in decaying rotating turbulence: Laboratory experiments and numerical simulations," Geophys. Astrophys. Fluid Dyn. 106, 557-573 (2012).

${ }^{23}$ G. Di Nitto, S. Espa, and A. Cenedese, "Simulating zonation in geophysical flows by laboratory experiments," Phys. Fluids 25, 086602 (2013).

${ }^{24}$ J. Pedlosky, Geophysical Fluid Dynamics, 2nd ed. (Springer-Verlag, 1987).

${ }^{25}$ Y. D. Afanasyev and J. Wells, "Quasi-two-dimensional turbulence on the polar beta-plane: Laboratory experiments," Geophys. Astrophys. Fluid Dyn. 99, 1-17 (2005).

${ }^{26}$ M. Duran-Matute, G. Di Nitto, R. R. Trieling, L. P. J. Kamp, and G. J. F. van Heijst, "The break-up of Ekman theory in a flow subjected to background rotation and driven by a non-conservative body force," Phys. Fluids 24, 116602 (2012).

${ }^{27} \mathrm{M}$. Moroni and A. Cenedese, "Comparison among feature tracking and more consolidated velocimetry image analysis techniques in a fully developed turbulent channel flow," Meas. Sci. Technol. 16, 2307 (2005).
${ }^{28}$ D. B. Chelton, M. G. Schlax, and R. M. Samelson, "Global observations of nonlinear mesoscale eddies," Prog. Oceanogr. 91, 167-216 (2011).

${ }^{29}$ N. A. Maximenko, B. Bang, and H. Sasaki, "Observational evidence of alternating zonal jets in the world ocean,” Geophys. Res. Lett. 32, L12607, https://doi.org/10.1029/2005GL022728 (2005).

${ }^{30}$ K. J. Richards, N. A. Maximenko, F. O. Bryan, and H. Sasaki, "Zonal jets in the Pacific Ocean," Geophys. Res. Lett. 33, L03605, https://doi.org/10.1029/2005GL02 4645 (2006).

${ }^{31}$ J. Sommeria, S. Meyers, and H. Swinney, "Experiments on vortices and Rossby waves in eastward and westward jets," in Nonlinear Topics in Ocean Physics, edited by A. Osborne (Elsevier, North-Holland, Amsterdam, 1991), pp. 227-269.

${ }^{32}$ J. Aubert, S. Jung, and H. L. Swinney, "Observations of zonal flow created by potential vorticity mixing in a rotating fluid," Geophys. Res. Lett. 29, 23-1-23-4, https://doi.org/10.1029/2002gl015422 (2002).

${ }^{33}$ R. H. Kraichnan, "Inertial ranges in two-dimensional turbulence," Phys. Fluids 10, 1417-1423 (1967).

${ }^{34}$ G. B. Arfken and H. J. Weber, Mathematical Methods for Physicists (Academic Press, 1999).

${ }^{35}$ A. Okubo, "Horizontal dispersion of floatable particles in the vicinity of velocity singularities such as convergences," Deep-Sea Res. 17, 445-454 (1970).

${ }^{36} \mathrm{~J}$. Weiss, "The dynamics of enstrophy transfer in two-dimensional hydrodynamics," Physica D 48, 273-294 (1991).

${ }^{37}$ D. Elhmaïdi, A. Provenzale, and A. Babiano, "Elementary topology of twodimensional turbulence from a Lagrangian viewpoint and single-particle dispersion," J. Fluid Mech. 257, 533-558 (1993).

${ }^{38}$ C. Pasquero, A. Provenzale, and A. Babiano, "Parameterization of dispersion in two-dimensional turbulence," J. Fluid Mech. 439, 279-303 (2001).

${ }^{39}$ J. Isern-Fontanet, E. García-Ladona, J. Font, and A. García-Olivares, "NonGaussian velocity probability density functions: An altimetric perspective of the Mediterranean sea," J. Phys. Oceanogr. 36, 2153-2164 (2006).

${ }^{40}$ E. M. Douglass and J. G. Richman, "Analysis of ageostrophy in strong surface eddies in the Atlantic Ocean," J. Geophys. Res.: Oceans 120, 1490-1507, https://doi.org/10.1002/2014jc010350 (2015).

${ }^{41}$ J. P. Boyd, "Planetary solitary waves," in Solitary Waves in Fluids (WIT Press, 2007), Chap. 6.

${ }^{42}$ G. K. Vallis, Atmospheric and Oceanic Fluid Dynamics: Fundamentals and Large-Scale Circulation (Cambridge University Press, Cambridge, 2017). 